\title{
Bifurcation analysis of a predator-prey system with sex-structure and sexual favoritism
}

\author{
Shunyi $\mathrm{Li}^{\mathrm{i}^{*}}$ and Zuoliang Xiong ${ }^{2}$
}

${ }^{*}$ Correspondence:

lishunyi19820425@163.com

'Department of Mathematics,

Qiannan Normal College for

Nationalities, Duyun, Guizhou 558000, China

Full list of author information is available at the end of the article

\section{Springer}

\begin{abstract}
In this paper, a predator-prey system with sex-structure and sexual favoritism is considered. Firstly, the impact of the sexual favoritism coefficient on the stability of the ordinary differential equation (ODE) model is studied. By choosing sexual favoritism coefficient as a bifurcation parameter, it is shown that a Hopf bifurcation can occur as it passes some critical value, and the stability of the bifurcation is also considered by using an analytical method. Secondly, the impact of the time delay on the stability of the delay differential equation (DDE) model is investigated, where time delay is regarded as a bifurcation parameter. It is found that a Hopf bifurcation can occur as the time delay passes some critical values. Using the normal form theory and center manifold argument, the explicit formulae which determine the stability, direction and other properties of bifurcating periodic solutions are derived. Numerical simulations are performed to support theoretical results and some complex dynamic behaviors are observed, including period-halving bifurcations, period-doubling bifurcations, high-order periodic oscillations, chaotic oscillation, fast-slow oscillation, even unbounded oscillation. Finally, a brief conclusion is given.
\end{abstract}

MSC: 34K13; 34K18; 34K60; 37D45; 37N25; 92D25

Keywords: predator-prey system; time delay; bifurcation; chaos; sex-structure; sexual favoritism

\section{Introduction and formulation of the model}

Sex ratio means the comparison of male and female individual number in populations. Usually, we assume the sex ratio is $1: 1$. However, to some wildlife, the sex ratio of populations will change with the kinds, mate, environment conditions, social behavior, resource, adaptability, heredity, gene structure, etc. (see [1-9]). The animal's sex ratio will change with different animals in the different life history stage. Along with the growth of the age, the male individuals tend to relatively decrease, but there exists a variety of birds for which, on the contrary, the male individuals relatively increase. In isolated populations, males compete locally for mates and resource, sex ratio will affect the dynamic behavior of the population [10]. Sex ratio is a basic dynamic factor for the analysis of populations, and it has important influence on the dynamic state of populations.

Based on the classical Lotka-Volterra model, Liu et al. [11] introduced the following sexstructure model:

$$
\left\{\begin{array}{l}
m^{\prime}(t)=b_{1} f(t)-d_{1} m(t)-k(m(t)+f(t)) m(t)-c_{1} m(t) x(t), \\
f^{\prime}(t)=f(t)\left(\beta-k(m(t)+f(t))-c_{1} x(t)\right), \\
x^{\prime}(t)=x(t)\left(-a-b x(t)+c_{2} m(t)+c_{2} f(t)\right),
\end{array}\right.
$$

C 2013 Li and Xiong; licensee Springer. This is an Open Access article distributed under the terms of the Creative Commons Attribution License (http://creativecommons.org/licenses/by/2.0), which permits unrestricted use, distribution, and reproduction in any medium, provided the original work is properly cited. 
where $m(t)$ and $f(t)$ are the male, female individuals of the prey population, $x(t)$ is the predator population. The parameters $a, b, b_{1}, b_{2}, c_{1}, c_{2}, d_{1}, d_{2}, k$ are positive, $b_{1}, b_{2}, d_{1}$, $d_{2}$ are constants of proportionality for male and female prey growth and death $\left(b_{2}>d_{2}\right.$, where $\left.\beta=b_{2}-d_{2}\right), a$ is the constants of proportionality for a predator, $c_{1}$ is the predation coefficient for a predator and $c_{1} / c_{2}\left(0<c_{1} / c_{2}<1\right)$ is the rate of conversing prey into a predator, respectively. The authors obtained the conditions for the equilibrium stability of system (1.1).

Moreover, Boukal et al. [12] considered sex-selective predation using several simple predator-prey models, for example, male-biased predation is frequently related to prey traits shaped by sexual selection, predators and parasitoids are attracted by mating signals of their male prey; female-biased predation is often related to prey traits shaped by fecundity selection since it is easier or more rewarding to detect them than prey. The author found that long-term effects of sex-selective predation depend on the interplay of predation bias and prey mating system, given the conclusion that 'predation on the "less limiting" prey sex can yield a stable predator-prey equilibrium, while predation on the other sex usually destabilizes the dynamics and promotes population collapses.' For the methods, models, data, results, and more details, see [12].

Considering system (1.1) with sexual favoritism (sex-selective predation), Liu et al. [11] introduced the following ODE model:

$$
\left\{\begin{array}{l}
m^{\prime}(t)=b_{1} f(t)-d_{1} m(t)-\sigma c_{1} m(t) x(t) \\
f^{\prime}(t)=f(t)\left(\beta-c_{1} x(t)\right) \\
x^{\prime}(t)=x(t)\left(-a+\sigma c_{2} m(t)+c_{2} f(t)\right)
\end{array}\right.
$$

where $\sigma$ is the sexual favoritism coefficient, $\sigma>1$ means that the predator prefers predating male prey to female prey, $0<\sigma<1$ means that the predator prefers predating female prey to male prey, and $\sigma=1$ means there is no sexual favoritism. The authors obtained the conditions for the equilibrium stability of system (1.2). But, how does the dynamic behavior go when the positive equilibrium loses stability? Does there exist a periodic solution or other rich dynamic behaviors?

Delays play an important role in the dynamics of populations. Delay can cause the loss of stability and can bifurcate various periodic solutions. Recently, there has been extensive work dealing with time delay systems (see [13-22]). In many processes of the real world, especially in many biological phenomena, the present dynamics, the present rate of change of the state variables depend not only on the present state of the processes but also on the history of the phenomenon, i.e., on past values of the state variables. We assume that the reproduction of the predator after predating the prey is not instantaneous and needs some discrete time delay required for gestation of the predator (see [23-25]). Then we formulate the following DDE model:

$$
\left\{\begin{array}{l}
m^{\prime}(t)=b_{1} f(t)-d_{1} m(t)-\sigma c_{1} m(t) x(t), \\
f^{\prime}(t)=f(t)\left(\beta-c_{1} x(t)\right), \\
x^{\prime}(t)=-a x(t)+\sigma c_{2} m(t-\tau) x(t-\tau)+c_{2} f(t-\tau) x(t-\tau),
\end{array}\right.
$$


where $\tau(\tau>0)$ is the time required for the gestation of the predator. The initial conditions for (1.3) are

$$
\left(\varphi_{1}(\theta), \varphi_{2}(\theta), \phi(\theta)\right) \in C_{+}=\left\{[-\tau, 0], \mathbb{R}_{+}^{3}\right\}, \quad \varphi_{1}(\theta)>0, \varphi_{2}(\theta)>0, \phi(\theta)>0,
$$

where $\mathbb{R}_{+}^{3}=\left\{(m, f, x) \in \mathbb{R}^{3}, m \geq 0, f \geq 0, x \geq 0\right\}$.

To the best of our knowledge, few papers focus on the predator-prey system with sexstructure. Recently, Xiong and Zhang [26] have studied a predator-prey model with sexstructure. They obtained the sufficient and realistic conditions for the existence of a positive periodic solution by constructing a $V$ functional, and using the result of the existence of positive periodic solutions, the global attractivity of a positive periodic solution was also obtained. Based on system (1.1), Li and Xiong [27] investigated the discrete periodic sex structure model and obtained sufficient and realistic conditions for the existence and global attractivity of a positive periodic solution for it. The pest management strategy of a prey-predator system model with sexual favoritism was considered by Pei et al. [28], and the conditions for a global asymptotically stable pest-eradication periodic solution and permanence of the system were established. The local asymptotic stability of system (1.2) has been studied by Liu et al. [11]. However, by choosing $\sigma$ as a bifurcation parameter, we obtain Hopf bifurcation conditions for system (1.2). In model (1.3), we introduce time delay due to the gestation of the predator. So, we believe that this is the first time that a predator-prey model with sex-structure and time delay has been formulated and analyzed.

This paper is organized as follows. In Section 2, we first focus on the stability of the equilibrium point and the Hopf bifurcation of ODE system (1.2) by choosing $\sigma$ as a bifurcation parameter. The stability of the bifurcation is also considered by using an analytical method introduced by Kazarinov [29]. In Section 3, we investigate the existence of Hopf bifurcations and the estimation of the length of delay to preserve the stability of DDE system (1.3). By using the normal form theory and center manifold argument introduced by Hassard [30], we derive the explicit formulae for determining the stability, direction, and other properties of bifurcating periodic solutions. Finally, in Section 4, numerical simulations are performed to support the theoretical results. Numerical results show that ODE system (1.2) considered has chaotic behavior under some parameter sets of values and the Hopf bifurcation of DDE system (1.3) is subcritical, and the bifurcating periodic solutions are unstable under certain conditions.

\section{ODE model (1.2)}

\subsection{Stability of equilibrium and the existence of a Hopf bifurcation}

Obviously, system (1.2) has one boundary equilibrium $E_{0}=(0,0,0)$ and a unique positive equilibrium

$$
E^{*}=\left(m^{*}, f^{*}, x^{*}\right)=\left(\frac{a b_{1}}{c_{2}\left(d_{1}+\sigma b_{1}+\sigma \beta\right)}, \frac{a\left(d_{1}+\sigma \beta\right)}{c_{2}\left(d_{1}+\sigma b_{1}+\sigma \beta\right)}, \frac{\beta}{c_{1}}\right) .
$$

Let $\bar{E}=(\bar{m}, \bar{f}, \bar{x})$ be any arbitrary equilibrium. Then the characteristic equation about $\bar{E}$ is given by

$$
\operatorname{det}(\bar{E})=\left|\begin{array}{ccc}
-d_{1}-\sigma c_{1} \bar{x}-\lambda & b_{1} & -\sigma c_{1} \bar{m} \\
0 & \beta-c_{1} \bar{x}-\lambda & -c_{1} \bar{f} \\
\sigma c_{2} \bar{x} & c_{2} \bar{x} & -a+\sigma c_{2} \bar{m}+c_{2} \bar{f}-\lambda
\end{array}\right|=0 .
$$


By verifying the characteristic roots of Eq. (2.1) at each equilibrium, it is easily seen that the equilibrium $E_{0}$ is always unstable.

Characteristic Eq. (2.1) about $E^{*}$ is given by

$$
H(\sigma)=\lambda^{3}+h_{2}(\sigma) \lambda^{2}+h_{1}(\sigma) \lambda+h_{0}(\sigma)
$$

where

$$
\begin{aligned}
& h_{2}(\sigma)=d_{1}+\sigma \beta>0, \quad h_{1}(\sigma)=\frac{a \beta\left(d_{1}+\sigma^{2} b_{1}+\sigma \beta\right)}{d_{1}+\sigma b_{1}+\sigma \beta}>0, \\
& h_{0}(\sigma)=a \beta\left(d_{1}+\sigma \beta\right)>0 .
\end{aligned}
$$

Let

$$
H_{1}(\sigma)=h_{1}(\sigma) h_{2}(\sigma)-h_{0}(\sigma)=\frac{\sigma a b_{1} \beta(\sigma-1)\left(d_{1}+\sigma \beta\right)}{d_{1}+\sigma b_{1}+\sigma \beta}
$$

obviously, $\sigma=\sigma_{0}=1$ is the unique positive root of $H_{1}(\sigma)=0$. By the Routh-Hurwitz criterion, $E^{*}$ is locally asymptotically stable if $\sigma>1$ and unstable if $0<\sigma<1$. Furthermore,

$$
\begin{aligned}
\left.\frac{\mathrm{d} H_{1}(\sigma)}{\mathrm{d} \sigma}\right|_{\sigma=\sigma_{0}} & =\left.\frac{a b_{1} \beta\left[\left(d_{1}+\sigma \beta\right)(2 \sigma-1)+\sigma \beta(\sigma-1)-\sigma\left(b_{1}+\beta\right)\left(d_{1}+\sigma \beta\right)(\sigma-1)\right]}{\left(d_{1}+\sigma b_{1}+\sigma \beta\right)^{2}}\right|_{\sigma=\sigma_{0}} \\
& =\frac{a b_{1} \beta\left(d_{1}+\beta\right)}{\left(d_{1}+b_{1}+\beta\right)}>0 .
\end{aligned}
$$

According to the above analysis and the result [31], we obtain the following theorem.

Theorem 2.1 The positive equilibrium $E^{*}$ is locally asymptotically stable when $\sigma>1$ and unstable when $0<\sigma<1$. There exists a critical value $\sigma_{0}=1$ such that a single Hopf bifurcation occurs at $\sigma=\sigma_{0}$ for decreasing $\sigma$, i.e., there exists a nontrivial orbitally periodic orbit of system (1.2) if $\sigma \in\left(\sigma_{0}-\varepsilon, \sigma_{0}\right)$.

\subsection{Hopf bifurcation analysis}

We deal with the Hopf bifurcation of system (1.2) using an analytical method introduced by Kazarinov [29]. We first introduce some definitions. Suppose that $\mathbb{C}^{n}$ is a linear space defined on the complex number field $\mathbb{C}$. For any vectors $x=\left(x_{1}, x_{2}, \ldots, x_{n}\right)^{T}$ and $y=\left(y_{1}, y_{2}, \ldots, y_{n}\right)^{T}$, where $x_{i}, y_{i} \in \mathbb{C}(i=1,2, \ldots, n),\langle x, y\rangle=\sum_{i=1}^{n} \bar{x}_{i} y_{i}$ is the inner product of the vectors $x$ and $y$.

Consider the following nonlinear system:

$$
x^{\prime}=A x+F(x), \quad x \in \mathbb{R}^{3},
$$

where $F(x)=O\left(\|x\|^{2}\right)$ is a smooth function, and it can be expanded into

$$
F(x)=\frac{1}{2} B(x, x)+\frac{1}{6} C(x, x, x)+O\left(\|x\|^{4}\right),
$$


where

$$
B_{i}(x, y)=\left.\sum_{j, k=1}^{3} \frac{\partial^{2} F_{i}(\xi)}{\partial \xi_{j} \partial \xi_{k}}\right|_{\xi=0} x_{j} y_{k}, \quad C_{j}(x, y, z)=\left.\sum_{j, k, l=1}^{3} \frac{\partial^{3} F_{i}(\xi)}{\partial \xi_{j} \partial \xi_{k} \partial \xi_{l}}\right|_{\xi=0} x_{j} y_{k} z_{l}
$$

for $i=1,2,3$. From (1.2) and (2.3), we have

$$
B(x, y)=\left(\begin{array}{c}
-c_{1}\left(x_{1} y_{3}+x_{3} y_{1}\right) \\
-c_{1}\left(x_{2} y_{3}+x_{3} y_{2}\right) \\
c_{2}\left(x_{1} y_{3}+x_{3} y_{1}+x_{2} y_{3}+x_{3} y_{2}\right)
\end{array}\right)
$$

and $\mathbb{C}(x, y, z)=(0,0,0)^{T}$. In Eq. (2.2), if the matrix $A$ only has a pair of pure imaginary eigenvalues $\lambda_{1,2}= \pm \alpha \mathrm{i}, \alpha>0$, and other eigenvalues are negative, there exists a single Hopf bifurcation. Let $q \in \mathbb{C}^{n}$ be a complex eigenvector corresponding to the eigenvalue $\lambda_{1}$, then we have $A q=\mathrm{i} \alpha q, A \bar{q}=-\mathrm{i} \alpha \bar{q}$. At the same time, we introduce the adjoint eigenvector $p \in$ $\mathbb{C}^{n}$ which satisfies the following conditions:

$$
A^{T} p=-\mathrm{i} \alpha p, \quad A^{T} \bar{p}=\mathrm{i} \alpha \bar{p}, \quad\langle p, q\rangle=1 .
$$

The two-dimensional center manifold can be parameterized by $w=\mathbb{R}^{2}=\mathbb{C}$, by means of $x=H(w, \bar{w})$, which is written as

$$
H(w, \bar{w})=w q+\bar{w} \bar{q}+\sum_{2 \leq j+k \leq 3} \frac{1}{j ! k !} h_{j k} w^{j} \bar{w}^{k}+O\left(|w|^{4}\right),
$$

with $h_{j k} \in \mathbb{C}^{3}, h_{j k}=\bar{h}_{k j}$.

Substituting these expressions into (2.2) and (2.3) one has

$$
H_{w}(w, \bar{w}) w^{\prime}+H_{\bar{w}}(w, \bar{w}) \bar{w}^{\prime}=F(H(w, \bar{w})) .
$$

The complex vectors $h_{i j}$ are to be determined so that Eq. (2.5) can be written as follows:

$$
w^{\prime}=\mathrm{i} \alpha w+\frac{1}{2} G_{21} w|w|^{2}+O\left(|w|^{4}\right),
$$

with $G_{21} \in \mathbb{C}$. Solving the linear system obtained by expanding (2.5), the coefficients of the quadratic terms of (2.2) lead to

$$
h_{11}=-A^{-1} B(q, \bar{q}), \quad h_{20}=\left(2 \mathrm{i} \alpha I_{3}-A\right)^{-1} B(q, q),
$$

where $I_{3}$ is the unit $3 \times 3$ matrix.

The coefficients of the cubic terms are also uniquely calculated, except for the term $w^{2} \bar{w}$, whose coefficient satisfies a singular system for

$$
\left(\mathrm{i} \alpha I_{3}-A\right) h_{21}=C(q, q, \bar{q})+B\left(\bar{q}, h_{20}\right)+2 B\left(q, h_{11}\right)-G_{21} q,
$$

which has a solution if and only if

$$
\left\langle p, C(q, q, \bar{q})+B\left(\bar{q}, h_{20}\right)+2 B\left(q, h_{11}\right)-G_{21} q\right\rangle=0 .
$$


Therefore

$$
G_{21}=\left\langle p, C(q, q, \bar{q})+B\left(\bar{q},\left(2 \mathrm{i} \alpha I_{3}-A\right)^{-1} B(q, q)\right)-2 B\left(q, A^{-1} B(q, \bar{q})\right)\right\rangle,
$$

and the first Lyapunov coefficient $l_{1}$, which decides, by the analysis of third-order terms at the equilibrium, its stability, if negative, or instability, if positive, is defined by

$$
l_{1}=\frac{1}{2 \alpha} \operatorname{Re} G_{21} .
$$

A Hopf point is called transversal if the curves of complex eigenvalues cross the imaginary axis with a non-zero derivative. In a neighborhood of a transversal Hopf point with $l_{1} \neq 0$, the dynamic behavior of system (1.2), reduced to the family of parameter-dependent continuations of the center manifold, is orbitally topologically equivalent to the complex normal form

$$
w^{\prime}=(\gamma+\mathrm{i} \alpha) w+l_{1} w|w|^{2},
$$

$w \in \mathbb{C}, \gamma, w, l_{1}$ are smooth continuations of $0, \alpha$ and the first Lyapunov coefficient at the Hopf point [28], respectively. When $l_{1}<0\left(l_{1}>0\right)$, a family of stable (unstable) periodic orbits can be found on this family of center manifolds which shrink to the equilibrium point at the Hopf point.

\section{DDE model (1.3)}

\subsection{Existence of a Hopf bifurcation}

In Section 2, we know that ODE system (1.2) has a unique positive equilibrium $E^{*}$. Then DDE system (1.3) also has a unique positive equilibrium $E^{*}$. The linearization of system (1.3) at the positive equilibrium $E^{*}$ is

$$
\left\{\begin{array}{l}
m^{\prime}(t)=-\left(d_{1}+\sigma c_{1} x^{*}\right) m(t)+b_{1} f(t)-\sigma c_{1} m^{*} x(t), \\
f^{\prime}(t)=-c_{1} f^{*} x(t), \\
x^{\prime}(t)=\sigma c_{2} x^{*} m(t-\tau)+c_{2} x^{*} f(t-\tau)+a(x(t-\tau)-x(t)) .
\end{array}\right.
$$

The associated characteristic equation of system (3.1) is

$$
\left|\begin{array}{ccc}
-d_{1}-\sigma \beta-\lambda & b_{1} & -\sigma c_{1} m^{\prime \prime} \\
0 & -\lambda & -c_{1} f^{\prime \prime} \\
\sigma c_{2} x^{*} e^{-\lambda \tau} & c_{2} x^{*} e^{-\lambda \tau} & a\left(e^{-\lambda \tau}-1\right)-\lambda
\end{array}\right|=0,
$$

that is,

$$
M(\lambda)+N(\lambda) e^{-\lambda \tau}=0
$$

where

$$
\begin{aligned}
& M(\lambda)=\lambda^{3}+m_{2} \lambda^{2}+m_{1} \lambda+m_{0}, \quad N(\lambda)=n_{2} \lambda^{2}+n_{1} \lambda+n_{0}, \\
& m_{2}=d_{1}+\sigma \beta+a>0, \quad n_{2}=-a<0,
\end{aligned}
$$




$$
\begin{aligned}
& m_{1}=a\left(d_{1}+\sigma \beta\right)>0, \quad n_{1}=-a\left(d_{1}+\sigma \beta\right)+\frac{a \beta\left(d_{1}+\sigma \beta+\sigma^{2} b_{1}\right)}{d_{1}+\sigma \beta+\sigma b_{1}}, \\
& m_{0}=0, \quad n_{0}=a \beta\left(d_{1}+\sigma \beta\right)>0 .
\end{aligned}
$$

From Section 2.1, we know that the equilibrium $E^{*}$ is locally asymptotically stable in the absence of delay if $\sigma \in(1, \infty)$. Suppose that $\lambda=\mathrm{i} \omega, \omega>0$, is a root of Eq. (3.3), and separating the real and imaginary parts, one can get that

$$
\left\{\begin{array}{l}
m_{2} \omega^{2}=\left(n_{0}-n_{2} \omega^{2}\right) \cos \omega \tau+n_{1} \omega \sin \omega \tau \\
\omega^{3}-m_{1} \omega=n_{1} \omega \cos \omega \tau-\left(n_{0}-n_{2} \omega^{2}\right) \sin \omega \tau
\end{array}\right.
$$

Adding up the squares of the corresponding sides of the above equations leads to

$$
\omega^{6}+p \omega^{4}+q \omega^{2}+r=0
$$

where

$$
\begin{aligned}
& p=m_{2}^{2}-2 m_{1}-n_{2}^{2}=\left(d_{1}+\sigma \beta\right)^{2}>0, \\
& q=m_{1}^{2}+2 n_{2} n_{0}-n_{1}^{2}, \\
& r=-n_{0}^{2}<0 .
\end{aligned}
$$

When $q>0$, from Eq. (3.6) we know that Eq. (3.5) has a unique positive root $\omega_{0}$. From Eq. (3.4) we have

$$
\cos \omega_{0} \tau=\frac{m_{2} \omega_{0}^{2}\left(n_{0}-n_{2} \omega_{0}^{2}\right)+n_{1} \omega_{0}\left(\omega_{0}^{3}-m_{1} \omega_{0}\right)}{\left(n_{0}-n_{2} \omega_{0}^{2}\right)^{2}+\left(n_{1} \omega_{0}\right)^{2}} .
$$

Thus,

$$
\tau_{n}=\frac{1}{\omega_{0}} \cos ^{-1}\left[\frac{m_{2} \omega_{0}^{2}\left(n_{0}-n_{2} \omega_{0}^{2}\right)+n_{1} \omega_{0}\left(\omega_{0}^{3}-m_{1} \omega_{0}\right)}{\left(n_{0}-n_{2} \omega_{0}^{2}\right)^{2}+\left(n_{1} \omega_{0}\right)^{2}}\right]+\frac{2 n \pi}{\omega_{0}}, \quad n=0,1,2, \ldots
$$

Let $\lambda(\tau)=v(\tau)+\mathrm{i} \omega(\tau)$ be the roots of Eq. (3.3) such that when $\tau=\tau_{n}$ satisfying $\nu\left(\tau_{n}\right)=0$ and $\omega\left(\tau_{n}\right)=\omega_{0}$, we can claim that $\left.\frac{\mathrm{d}(\operatorname{Re} \lambda)}{\mathrm{d} \tau}\right|_{\tau=\tau_{n}}>0$. In fact, differentiating both sides of (3.3) with respect to $\tau$, we get

$$
\begin{aligned}
& {\left[\left(3 \lambda^{2}+2 m_{2} \lambda+m_{1}\right)+\left(2 n_{2} \lambda+n_{1}\right) e^{-\lambda \tau}-\tau\left(n_{2} \lambda^{2}+n_{1} \lambda+n_{0}\right) e^{-\lambda \tau}\right] \frac{\mathrm{d} \lambda}{\mathrm{d} \tau}} \\
& \quad=\lambda\left(n_{2} \lambda^{2}+n_{1} \lambda+n_{0}\right) e^{-\lambda} \tau,
\end{aligned}
$$

then

$$
\begin{aligned}
\left(\frac{\mathrm{d} \lambda}{\mathrm{d} \tau}\right)^{-1} & =\frac{3 \lambda^{2}+2 m_{2} \lambda+m_{1}}{\lambda\left(n_{2} \lambda^{2}+n_{1} \lambda+n_{0}\right) e^{-\lambda \tau}}+\frac{2 n_{2} \lambda+n_{1}}{\lambda\left(n_{2} \lambda^{2}+n_{1} \lambda+n_{0}\right)}-\frac{\tau}{\lambda} \\
& =\frac{3 \lambda^{2}+2 m_{2} \lambda+m_{1}}{-\lambda^{2}\left(\lambda^{2}+m_{2} \lambda+m_{1}\right)}+\frac{2 n_{2} \lambda+n_{1}}{\lambda\left(n_{2} \lambda^{2}+n_{1} \lambda+n_{0}\right)}-\frac{\tau}{\lambda} \\
& =\frac{2 \lambda+m_{2}}{-\lambda\left(\lambda^{2}+m_{2} \lambda+m_{1}\right)}+\frac{n_{2} \lambda^{2}-n_{0}}{\lambda^{2}\left(n_{2} \lambda^{2}+n_{1} \lambda+n_{0}\right)}-\frac{\tau}{\lambda}
\end{aligned}
$$


Therefore,

$$
\begin{aligned}
\operatorname{sign} & {\left[\frac{\mathrm{d}(\operatorname{Re} \lambda)}{\mathrm{d} \tau}\right]_{\lambda=\mathrm{i} \omega_{0}} } \\
= & \operatorname{sign}\left[\operatorname{Re}\left(\frac{\mathrm{d} \lambda}{\mathrm{d} \tau}\right)^{-1}\right]_{\lambda=\mathrm{i} \omega_{0}} \\
= & \operatorname{sign}\left\{\operatorname{Re}\left[\frac{2 \lambda+m_{2}}{-\lambda\left(\lambda^{2}+m_{2} \lambda+m_{1}\right)}+\frac{n_{2} \lambda^{2}-n_{0}}{\lambda^{2}\left(n_{2} \lambda^{2}+n_{1} \lambda+n_{0}\right)}-\frac{\tau}{\lambda}\right]\right\}_{\lambda=\mathrm{i} \omega_{0}} \\
= & \frac{1}{\omega_{0}^{2}} \operatorname{sign}\left\{\operatorname{Re}\left[\frac{\omega_{0}^{2}\left(m_{2}+2 \omega_{0} \mathrm{i}\right)}{m_{2} \omega_{0}^{2}+\left(\omega_{0}^{3}-m_{1} \omega_{0}\right) \mathrm{i}}+\frac{n_{2} \omega_{0}^{2}+n_{0}}{\left(n_{0}-n_{2} \omega_{0}^{2}\right)+n_{1} \omega_{0} \mathrm{i}}\right]\right\} \\
= & \frac{1}{\omega_{0}^{2}} \operatorname{sign}\left[\frac{\left(m_{2} \omega_{0}^{2}-m_{0}\right)\left(m_{0}+m_{2} \omega_{0}^{2}\right)+2 \omega_{0}^{3}\left(\omega_{0}^{3}-m_{1} \omega_{0}\right)}{\left(m_{2} \omega_{0}^{2}-m_{0}\right)^{2}+\left(\omega_{0}^{3}-m_{1} \omega_{0}\right)^{2}}\right. \\
& \left.+\frac{\left(n_{0}-n_{2} \omega_{0}^{2}\right)\left(n_{2} \omega_{0}^{2}+n_{0}\right)}{\left(n_{0}-n_{2} \omega_{0}^{2}\right)^{2}+\left(n_{1} \omega_{0}\right)^{2}}\right] \\
= & \frac{1}{\Gamma} \operatorname{sign}\left[m_{2}^{2} \omega_{0}^{4}+2 \omega_{0}^{3}\left(\omega_{0}^{3}-m_{1} \omega_{0}\right)+\left(n_{0}-n_{2} \omega_{0}^{2}\right)\left(n_{2} \omega_{0}^{2}+n_{0}\right)\right] \\
= & \frac{1}{\Gamma} \operatorname{sign}\left[2 \omega_{0}^{6}+\left(m_{2}^{2}-2 m_{1}-n_{2}^{2}\right) \omega_{0}^{4}+n_{0}^{2}\right] \\
= & \frac{1}{\Gamma} \operatorname{sign}\left[2 \omega_{0}^{6}+p \omega_{0}^{4}+n_{0}^{2}\right],
\end{aligned}
$$

where $\Gamma=\omega_{0}^{2}\left[\left(n_{0}-n_{2} \omega_{0}^{2}\right)^{2}+\left(n_{1} \omega_{0}\right)^{2}\right]>0$. According to the Hopf bifurcation theorem for functional differential equations [32], we have the following result.

Theorem 3.1 Suppose that $\sigma \in(1, \infty)$. (i) There exists a $\tau_{0}$ such that for $\tau \in\left[0, \tau_{0}\right)$ the positive equilibrium $E^{*}$ of system (1.3) is asymptotically stable and unstable when $\tau>\tau_{0}$.

(ii) System (1.3) can undergo a Hopf bifurcation at the positive equilibrium $E^{*}$ when $\tau=\tau_{n}$ $(n=0,1,2, \ldots)$, where $\tau_{n}$ is defined by (3.7).

Remark 3.1 It must be pointed out that Theorem 3.1 cannot determine the stability and the direction of bifurcating periodic solutions, that is, the periodic solutions may exist either for $\tau>\tau_{0}$ or for $\tau<\tau_{0}$, near $\tau_{0}$. Furthermore, we can investigate the stability of the bifurcating periodic orbits by analyzing higher-order terms according to Hassard et al. [30] by using normal form theory and center manifold theorem and prove that the Hopf bifurcation is subcritical and bifurcating periodic solutions are unstable.

\subsection{Estimation of the length of delay to preserve stability}

We consider system (1.3) and the space of all real-valued continuous functions defined on $[-\tau, \infty)$ satisfying the initial conditions on $[-\tau, 0]$. Taking Laplace transform of the system given by (3.1)

$$
\left\{\begin{array}{c}
\left(s+d_{1}+\sigma c_{1} x^{*}\right) \bar{m}(s)=b_{1} \bar{f}(s)-\sigma c_{1} m^{*} \bar{x}(s)+m(0) \\
s \bar{f}(s)=-c_{1} f^{*} \bar{x}(s)+f(0) \\
(s+a) \bar{x}(s)=e^{-s \tau} \sigma c_{2} x^{*}\left(\bar{m}(s)+K_{1}(s)\right)+e^{-s \tau} c_{2} x^{*}\left(\bar{f}(s)+K_{2}(s)\right) \\
\quad+e^{-s \tau} a\left(\bar{x}(s)+K_{3}(s)\right)+x(0),
\end{array}\right.
$$


where

$$
K_{1}(s)=\int_{-\tau}^{0} e^{-s t} m(t) \mathrm{d} t, \quad K_{2}(s)=\int_{-\tau}^{0} e^{-s t} f(t) \mathrm{d} t, \quad K_{3}(s)=\int_{-\tau}^{0} e^{-s t} x(t) \mathrm{d} t,
$$

and $\bar{m}(s), \bar{f}(s), \bar{x}(s)$ are the Laplace transforms of $m(t), f(t), x(t)$, respectively.

Following along the lines of [33] and using Nyquist criterion [34], it can be shown that the conditions of local asymptotic stability of $E^{*}$ given by [34] are

$$
\begin{aligned}
& \operatorname{Im} H\left(\mathrm{i} \eta_{0}\right)>0, \\
& \operatorname{Re} H\left(\mathrm{i} \eta_{0}\right)=0,
\end{aligned}
$$

where $H(\lambda)=M(\lambda)+N(\lambda) e^{-\lambda \tau}=0$, and $\eta_{0}$ is the smallest positive root of Eq. (3.10).

We have already shown that $E^{*}$ is stable in the absence of delay when $\sigma \in(1, \infty)$. Hence, by continuity, all eigenvalues will continue to have negative real parts for sufficiently small $\tau>0$ provided one can guarantee that no eigenvalues with positive real parts bifurcate from infinity as $\tau$ increases from zero. This can be proved using Butler's lemma [34], already stated before. In fact, Eqs. (3.9) and (3.10) give

$$
\begin{aligned}
& m_{1} \eta_{0}-\eta_{0}^{3}>-n_{1} \eta_{0} \cos \eta_{0} \tau+\left(n_{0}-n_{2} \eta_{0}^{2}\right) \sin \eta_{0} \tau, \\
& m_{2} \eta_{0}^{2}=\left(n_{0}-n_{2} \eta_{0}^{2}\right) \cos \eta_{0} \tau+n_{1} \eta_{0} \sin \eta_{0} \tau .
\end{aligned}
$$

Equations (3.11) and (3.12), if satisfied simultaneously, are sufficient conditions to guarantee stability. We shall utilize them to get an estimate on the length of delay. Our aim is to find an upper bound $\eta_{+}$on $\eta_{0}$, independent of $\tau$, and then to estimate $\tau$ so that (3.11) holds for all values of $\eta, 0 \leq \eta \leq \eta_{+}$, and in particular at $\eta=\eta_{0}$.

Maximizing the right-hand side of Eq. (3.12) subject to $\left|\sin \eta_{0} \tau\right| \leq 1,\left|\cos \eta_{0} \tau\right| \leq 1$, we obtain

$$
m_{2} \eta_{0}^{2} \leq n_{0}-n_{2} \eta_{0}^{2}+\left|n_{1}\right| \eta_{0} \quad\left(n_{2}<0\right)
$$

Hence, if

$$
\eta_{+}=\frac{\left|n_{1}\right|+\sqrt{n_{1}^{2}+4 n_{0}\left(m_{2}+n_{2}\right)}}{2\left(m_{2}+n_{2}\right)}
$$

then, clearly, from (3.13) we have $\eta_{0} \leq \eta_{+}$.

From inequality (3.11) we obtain

$$
\eta_{0}^{2}<m_{1}+n_{1} \cos \eta_{0} \tau-n_{0} \frac{\sin \eta_{0} \tau}{\eta_{0}}+n_{2} \eta_{0} \sin \eta_{0} \tau
$$

As $E^{*}$ is locally asymptotically stable for $\tau=0$, therefore, for sufficiently small $\tau>0$, (3.15) will continue to hold. Substituting (3.12) in (3.15) and rearranging, we get

$$
\begin{aligned}
& \left(n_{0}-n_{2} \eta_{0}^{2}-m_{2} n_{1}\right)\left(\cos \eta_{0} \tau-1\right)+\left[\left(n_{1}-m_{2} n_{2}\right) \eta_{0}+\frac{m_{2} n_{0}}{\eta_{0}}\right] \sin \eta_{0} \tau \\
& \quad<m_{2}\left(m_{1}+n_{1}\right)-n_{0} n_{2}^{2} \eta_{0} .
\end{aligned}
$$


Using the bounds

$$
\left\{\begin{array}{l}
\left(n_{0}-n_{2} \eta_{0}^{2}-m_{2} n_{1}\right)\left(\cos \eta_{0} \tau-1\right) \leq \frac{1}{2} \eta_{+}^{2} \tau^{2}\left|n_{0}-n_{2} \eta_{+}^{2}-m_{2} n_{1}\right| \\
{\left[\left(n_{1}-m_{2} n_{2}\right) \eta_{0}+\frac{m_{2} n_{0}}{\eta_{0}}\right] \sin \eta_{0} \tau \leq\left[\left(n_{1}-m_{2} n_{2}\right) \eta_{+}^{2}+m_{2} n_{0}\right] \tau}
\end{array}\right.
$$

(it can be easily shown that $n_{1}-m_{2} n_{2}$ is positive), we obtain from (3.16)

$$
Q_{1} \tau^{2}+Q_{2} \tau<Q_{3}
$$

where

$$
\begin{aligned}
& Q_{1}=\frac{1}{2} \eta_{+}^{2}\left|n_{0}-n_{2} \eta_{+}^{2}-m_{2} n_{1}\right|, \quad Q_{2}=\left[\left(n_{1}-m_{2} n_{2}\right) \eta_{+}^{2}+m_{2} n_{0}\right], \\
& Q_{3}=m_{2}\left(m_{1}+n_{1}\right)-n_{0}+n_{2}^{2} \eta_{0} .
\end{aligned}
$$

Hence, if

$$
\tau_{+}=\frac{\sqrt{Q_{2}^{2}+4 Q_{1} Q_{3}}-Q_{2}}{2 Q_{1}},
$$

then stability is preserved for $0 \leq \tau \leq \tau_{+}$.

\subsection{Direction and stability of a Hopf bifurcation}

In Section 3.1, we have obtained the conditions under which a family of periodic solutions bifurcates from the positive equilibrium of system (1.3) when the delay crosses through the critical values $\tau_{n}$. In this subsection, we shall study the direction of these Hopf bifurcations and the stability of bifurcated periodic solutions arising through Hopf bifurcations by applying the normal form theory and center manifold theorem introduced by Hassard et al. [30].

Let $u_{1}=m(\tau t), u_{2}=f(\tau t), u_{3}=x(\tau t), \tau=\tau_{n}+\mu$, where $\tau_{n}$ is defined by $(3.7), \mu \in \mathbb{R}$, then system (1.3) can be transformed as an FDE in $C=C\left([-1,0], \mathbb{R}^{3}\right)$.

$$
u^{\prime}=L_{\mu}\left(u_{t}\right)+H\left(\mu, u_{t}\right)
$$

where $u(t)=\left(u_{1}, u_{2}, u_{3}\right)^{T} \in \mathbb{R}^{3}$ and $L_{\mu}: C \rightarrow \mathbb{R}, H: \mathbb{R} \times C \rightarrow \mathbb{R}$ are given respectively by

$$
\begin{aligned}
L_{\mu}(\varphi)= & \left(\tau_{n}+\mu\right)\left(\begin{array}{ccc}
-d_{1}-\sigma \beta & b_{1} & -\sigma c_{1} m^{*} \\
0 & 0 & -c_{1} f^{*} \\
0 & 0 & -a
\end{array}\right)\left(\begin{array}{l}
\varphi_{1}(0) \\
\varphi_{2}(0) \\
\varphi_{3}(0)
\end{array}\right) \\
& +\left(\tau_{n}+\mu\right)\left(\begin{array}{ccc}
0 & 0 & 0 \\
0 & 0 & 0 \\
\sigma c_{2} x^{*} & c_{2} x^{*} & a
\end{array}\right)\left(\begin{array}{l}
\varphi_{1}(-1) \\
\varphi_{2}(-1) \\
\varphi_{3}(-1)
\end{array}\right)
\end{aligned}
$$

and

$$
H(\mu, \phi)=\left(\tau_{n}+\mu\right)\left(\begin{array}{c}
-\sigma c_{1} \phi_{1}(0) \phi_{3}(0) \\
-c_{1} \phi_{2}(0) \phi_{3}(0) \\
\sigma c_{2} \phi_{1}(-1) \phi_{3}(-1)+c_{2} \phi_{2}(-1) \phi_{3}(-1)
\end{array}\right) .
$$


By the Riesz representation theorem, there exists a matrix whose components are bounded variation functions $\eta(\theta, \mu)$ in $[-1,0]$ such that

$$
L_{\mu} \phi=\int_{-1}^{0} \mathrm{~d} \eta(\theta, \mu) \phi(\theta), \quad \phi \in C\left([-1,0], \mathbb{R}^{3}\right) .
$$

In fact, we choose

$$
\begin{aligned}
\eta(\theta, \mu)= & \left(\tau_{n}+\mu\right)\left(\begin{array}{ccc}
-d_{1}-\sigma \beta & b_{1} & -\sigma c_{1} m^{*} \\
0 & 0 & -c_{1} f^{*} \\
0 & 0 & -a
\end{array}\right) \delta(\theta) \\
& -\left(\tau_{n}+\mu\right)\left(\begin{array}{ccc}
0 & 0 & 0 \\
0 & 0 & 0 \\
\sigma c_{2} x^{*} & c_{2} x^{*} & a
\end{array}\right) \delta(\theta+1),
\end{aligned}
$$

where $\delta(\theta)$ is a Dirac function, then (3.20) is satisfied.

For $\phi \in C^{1}\left([-1,0], \mathbb{R}^{3}\right)$, define

$$
A(\mu) \phi= \begin{cases}\frac{\mathrm{d} \phi(\theta)}{\mathrm{d} \theta}, & -1 \leq \theta<0 \\ \int_{-1}^{0} \mathrm{~d} \eta(\mu, s) \phi(s), & \theta=0\end{cases}
$$

and

$$
R(\mu) \phi= \begin{cases}0, & -1 \leq \theta<0, \\ H(\mu, \phi), & \theta=0 .\end{cases}
$$

Then system (3.17) can be transformed into an operator differential equation of the form

$$
u_{t}^{\prime}=A(\mu) u_{t}+R(\mu) u_{t}
$$

where $u_{t}=u(t+\theta), \theta \in[-1,0]$. The adjoint operator $A^{*}$ of $A$ is defined by

$$
A^{*}(\mu) \psi= \begin{cases}-\frac{\mathrm{d} \psi(s)}{\mathrm{d} s}, & 0<s \leq 1 \\ \int_{-1}^{0} \psi(-t) \mathrm{d} \eta(0, t), & s=0\end{cases}
$$

associated with a bilinear form

$$
\langle\psi(s), \phi(s)\rangle=\bar{\psi}(0) \phi(0)-\int_{\theta=-1}^{0} \int_{\xi=0}^{\theta} \bar{\psi}^{T}(\xi-\theta) \mathrm{d} \eta(\theta) \phi(\xi) \mathrm{d} \xi,
$$

where $\eta(\theta)=\eta(\theta, 0)$, we know that $\pm \mathbf{i} \tau_{n} \omega_{0}$ are eigenvalues of $A(0)$. Thus they are also eigenvalues of $A^{*}$. To determine the Poincaré normal form of the operator $A$, we need to calculate the eigenvector $q$ of $A$ belonging to the eigenvalue $i \omega_{0}$ and the eigenvector $q^{\prime \prime}$ of $A^{*}$ belonging to the eigenvalue $-\mathrm{i} \omega_{0}$.

Suppose that $q(\theta)=\left(1, \rho_{1}, \rho_{2}\right)^{T} e^{\mathrm{i} \omega_{0} \theta}$ is the eigenvector of $A(0)$ corresponding to $\mathrm{i} \omega_{0}$. Then $A(0) q(\theta)=\mathrm{i} \omega_{0} q(\theta)$. It follows from the definition of $A(0),(3.20)$ and (3.21) that

$$
\left(\begin{array}{ccc}
-d_{1}-\sigma \beta-\mathrm{i} \omega_{0} & b_{1} & -\sigma c_{1} m^{\prime \prime} \\
0 & -\mathrm{i} \omega_{0} & -c_{1} f^{*} \\
\sigma c_{2} x^{*} e^{-\mathrm{i} \omega_{0} \tau_{n}} & c_{2} x^{*} e^{-\mathrm{i} \omega_{0} \tau_{n}} & a\left(e^{-\mathrm{i} \omega_{0} \tau_{n}}-1\right)-\mathrm{i} \omega_{0}
\end{array}\right) q(0)=\left(\begin{array}{l}
0 \\
0 \\
0
\end{array}\right) .
$$


We, therefore, derive that

$$
q(0)=\left(1, \rho_{1}, \rho_{2}\right)^{T}=\left(1, \frac{f^{*}\left(d_{1}+\sigma \beta+\mathrm{i} \omega_{0}\right)}{b_{1} f^{*}+\mathrm{i} \omega_{0} \sigma m^{*}}, \frac{-\mathrm{i} \omega_{0}\left(d_{1}+\sigma \beta+\mathrm{i} \omega_{0}\right)}{c_{1}\left(b_{1} f^{*}+\mathrm{i} \omega_{0} \sigma m^{*}\right)}\right)^{T} .
$$

On the other hand, suppose that $q^{*}(s)=D\left(1, \alpha_{1}, \alpha_{2}\right) e^{\mathrm{i} \omega_{0} s}$ is the eigenvector of $A^{*}(0)$ corresponding to $-\mathrm{i} \omega_{0}$. Similarly, we can get

$$
q^{*}(0)=D\left(1, \alpha_{1}, \alpha_{2}\right)=D\left(1, \frac{\mathrm{i} \omega_{0}-\left(d_{1}+\sigma \beta+\sigma b_{1}\right)}{\mathrm{i} \omega_{0} \sigma}, \frac{d_{1}+\sigma \beta-\mathrm{i} \omega_{0}}{\sigma c_{2} x^{*} e^{\mathrm{i} \omega_{0} \tau_{n}}}\right) .
$$

In order to assure $\left\langle q^{*}(s), q(\theta)\right\rangle=1$, we need to determine the value of $D$. From (3.26), we have

$$
\begin{aligned}
& \left\langle q^{*}(s), q(\theta)\right\rangle \\
& \quad=\bar{D}\left\{\left(1, \bar{\alpha}_{1}, \bar{\alpha}_{2}\right)\left(1, \rho_{1}, \rho_{2}\right)^{T}-\int_{\theta=-1}^{0} \int_{\xi=0}^{\theta}\left(1, \bar{\alpha}_{1}, \bar{\alpha}_{2}\right) e^{-\mathrm{i}(\xi-\theta)} \mathrm{d} \eta(\theta)\left(1, \rho_{1}, \rho_{2}\right)^{T} e^{\mathrm{i} \xi \omega_{0}} \mathrm{~d} \xi\right\} \\
& \quad=\bar{D}\left\{1+\bar{\alpha}_{1} \rho_{1}+\bar{\alpha}_{2} \rho_{2}-\int_{-1}^{0}\left(1, \bar{\alpha}_{1}, \bar{\alpha}_{2}\right) \theta e^{\mathrm{i} \omega_{0} \theta} \mathrm{d} \eta(\theta)\left(1, \rho_{1}, \rho_{2}\right)^{T}\right\} \\
& \quad=\bar{D}\left\{1+\bar{\alpha}_{1} \rho_{1}+\bar{\alpha}_{2} \rho_{2}+\tau_{n} e^{-\mathrm{i} \omega_{0} \tau_{n}}\left[c_{2} x^{*}\left(\sigma+\bar{\alpha}_{1} \rho_{1}\right)+a \bar{\alpha}_{2} \rho_{2}\right]\right\} .
\end{aligned}
$$

Thus, we can choose

$$
D=\left\{1+\alpha_{1} \bar{\rho}_{1}+\alpha_{2} \bar{\rho}_{2}+\tau_{n} e^{\mathrm{i} \omega_{0} \tau_{n}}\left[c_{2} x^{*}\left(\sigma+\alpha_{1} \bar{\rho}_{1}\right)+a \alpha_{2} \bar{\rho}_{2}\right]\right\}^{-1}
$$

Let $u_{t}$ be the solution of Eq. (3.17) when $\mu=0$ and define

$$
z(t)=\left\langle q^{*}, u_{t}\right\rangle, \quad W(t, \theta)=u_{t}(\theta)-z(t) q(\theta)-\bar{z}(t) q^{*}(\theta)=u_{t}(\theta)-2 \operatorname{Re}\{z(t) q(\theta)\} .
$$

On the center manifold $C_{0}$, we have

$$
W(t, \theta)=W(z(t), \bar{z}(t), \theta),
$$

where

$$
W(z(t), \bar{z}(t), \theta)=W_{20}(\theta) \frac{z^{2}}{2}+W_{11} z \bar{z}+W_{02} \frac{\bar{z}^{2}}{2}+\cdots
$$

$z$ and $\bar{z}$ are local coordinates of the center manifold $C_{0}$ in the direction of $q$ and $q^{*}$, respectively. For the solution $u_{t} \in C_{0}$, since $\mu=0$, we have

$$
\begin{aligned}
\dot{z} & =\mathrm{i} \omega_{0} z+\left\langle\bar{q}^{*}(\theta), H(0, W(z, \bar{z}, \theta)+2 \operatorname{Re}\{z q(\theta)\})\right\rangle \\
& =\mathrm{i} \omega_{0} z+\bar{q}^{*}(\theta) H(0, W(z, \bar{z}, \theta)+2 \operatorname{Re}\{z q(\theta)\}) \\
& =\mathrm{i} \omega_{0} z+\bar{q}^{*}(0) H(0, W(z, \bar{z}, 0)+2 \operatorname{Re}\{z q(0)\}) \\
& \stackrel{\text { def }}{=} \mathrm{i} \omega_{0} z+\bar{q}^{*}(0) H_{0}(z, \bar{z}) .
\end{aligned}
$$


We rewrite $(3.28)$ as $\dot{z}=\mathrm{i} \omega_{0} z+g(z, \bar{z})$ with

$$
g(z, \bar{z})=q^{*}(0) H_{0}(z, \bar{z})=g_{20} \frac{z^{2}}{2}+g_{11} z \bar{z}+g_{02} \frac{\bar{z}^{2}}{2}+g_{21} \frac{z^{2} \bar{z}}{2}+\cdots
$$

Noting that $u_{t}(\theta)=\left(u_{1 t}(\theta), u_{2 t}(\theta), u_{3 t}(\theta)\right)=W(t, \theta)+z q(\theta)+\bar{z} \bar{q}(\theta)$, it follows

$$
\begin{aligned}
& g(z, \bar{z})=\bar{q}^{*}(0) H_{0}(z, \bar{z}) \\
& =\bar{D} \tau_{n}\left(1, \bar{\alpha}_{1}, \bar{\alpha}_{2}\right)\left(\begin{array}{c}
-\sigma c_{1} \phi_{1}(0) \phi_{3}(0) \\
-c_{1} \phi_{2}(0) \phi_{3}(0) \\
-\sigma c_{2} \phi_{1}(-1) \phi_{3}(-1)+c_{2} \phi_{2}(-1) \phi_{3}(-1)
\end{array}\right) \\
& =\bar{D} \tau_{n}\left\{\left[-c_{1} \rho_{2}\left(\sigma+\bar{\alpha} \rho_{1}\right)+c_{2} \alpha_{2} \bar{\alpha}_{2} e^{-2 \mathrm{i} \omega_{0} \tau_{n}}\left(\sigma+\alpha_{1}\right)\right] z^{2}\right. \\
& +\left[-\sigma\left(\rho_{2}+\bar{\rho}_{2}\right)-\bar{\alpha}_{1} c_{1}\left(\bar{\rho}_{1} \rho_{2}+\rho_{1} \bar{\rho}_{2}\right)+\bar{\alpha}_{2} c_{2}\left(\sigma\left(\alpha_{2}+\bar{\alpha}_{2}\right)+\alpha_{1} \bar{\alpha}_{2}+\bar{\alpha}_{1} \alpha_{2}\right)\right] z \bar{z} \\
& +\left[-c_{1} \bar{\rho}_{2}\left(\sigma+\bar{\alpha} \bar{\rho}_{1}\right)+c_{2} \bar{\alpha}_{2}^{2} e^{2 \mathrm{i} \omega_{0} \tau_{n}}\left(\sigma+\bar{\alpha}_{1}\right)\right] \bar{z}^{2} \\
& +\left\{-\sigma c_{1}\left[W_{11}^{(2)}(0)+\frac{1}{2} W_{20}^{(2)}(0)+\frac{1}{2} W_{20}^{(1)}(0) \bar{\rho}_{1}+W_{11}^{(1)}(0) \rho_{1}\right]\right. \\
& -\bar{\alpha}_{1} c_{1}\left[W_{11}^{(3)}(0) \bar{\rho}_{1}+\frac{1}{2} W_{20}^{(3)}(0) \bar{\rho}_{1}+\frac{1}{2} W_{20}^{(2)}(0) \bar{\rho}_{2}+W_{11}^{(2)}(0) \rho_{2}\right] \\
& +\bar{\alpha}_{2} c_{2}\left[W_{11}^{(3)}(-1) e^{-\mathrm{i} \omega_{0} \tau_{n}}\left(\sigma+\alpha_{1}\right)+\frac{1}{2} W_{20}^{(3)}(-1) e^{\mathrm{i} \omega_{0} \tau_{n}}\left(\sigma+\bar{\alpha}_{1}\right)\right. \\
& +\frac{1}{2} \bar{\alpha}_{2} e^{\mathrm{i} \omega_{0} \tau_{n}}\left(\sigma W_{20}^{(1)}(-1)+W_{20}^{(2)}(-1)\right) \\
& \left.\left.\left.+\alpha_{2} e^{-\mathrm{i} \omega_{0} \tau_{n}}\left(\sigma W_{11}^{(1)}(-1)+W_{11}^{(2)}(-1)\right)\right]\right\} z^{2} \bar{z}\right\} \text {. }
\end{aligned}
$$

Comparing the coefficients with (3.29), we have

$$
\begin{aligned}
g_{20}= & 2 \bar{D} \tau_{n}\left[-c_{1} \rho_{2}\left(\sigma+\bar{\alpha} \rho_{1}\right)+c_{2} \alpha_{2} \bar{\alpha}_{2} e^{-2 \mathrm{i} \omega_{0} \tau_{n}}\left(\sigma+\alpha_{1}\right)\right] \\
g_{11}= & \bar{D} \tau_{n}\left[-\sigma\left(\rho_{2}+\bar{\rho}_{2}\right)-\bar{\alpha}_{1} c_{1}\left(\bar{\rho}_{1} \rho_{2}+\rho_{1} \bar{\rho}_{2}\right)+\bar{\alpha}_{2} c_{2}\left(\sigma\left(\alpha_{2}+\bar{\alpha}_{2}\right)+\alpha_{1} \bar{\alpha}_{2}+\bar{\alpha}_{1} \alpha_{2}\right)\right], \\
g_{02}= & 2 \bar{D} \tau_{n}\left[-c_{1} \bar{\rho}_{2}\left(\sigma+\bar{\alpha}_{1} \bar{\rho}_{1}\right)+c_{2} \bar{\alpha}_{2}^{2} e^{2 \mathrm{i} \omega_{0} \tau_{n}}\left(\sigma+\bar{\alpha}_{1}\right)\right], \\
g_{21}= & 2 \bar{D} \tau_{n}\left\{-\sigma c_{1}\left[W_{11}^{(2)}(0)+\frac{1}{2} W_{20}^{(2)}(0)+\frac{1}{2} W_{20}^{(1)}(0) \bar{\rho}_{1}+W_{11}^{(1)}(0) \rho_{1}\right]\right. \\
& -\bar{\alpha}_{1} c_{1}\left[W_{11}^{(3)}(0) \bar{\rho}_{1}+\frac{1}{2} W_{20}^{(3)}(0) \bar{\rho}_{1}+\frac{1}{2} W_{20}^{(2)}(0) \bar{\rho}_{2}+W_{11}^{(2)}(0) \rho_{2}\right] \\
& +\bar{\alpha}_{2} c_{2}\left[W_{11}^{(3)}(-1) e^{-\mathrm{i} \omega_{0} \tau_{n}}\left(\sigma+\alpha_{1}\right)+\frac{1}{2} W_{20}^{(3)}(-1) e^{\mathrm{i} \omega_{0} \tau_{n}}\left(\sigma+\bar{\alpha}_{1}\right)\right. \\
& +\frac{1}{2} \bar{\alpha}_{2} e^{\mathrm{i} \omega_{0} \tau_{n}}\left(\sigma W_{20}^{(1)}(-1)+W_{20}^{(2)}(-1)\right) \\
& \left.\left.+\alpha_{2} e^{-\mathrm{i} \omega_{0} \tau_{n}}\left(\sigma W_{11}^{(1)}(-1)+W_{11}^{(2)}(-1)\right)\right]\right\} .
\end{aligned}
$$


In order to determine $g_{21}$, we focus on the computation of $W_{20}(\theta)$ and $W_{11}(\theta)$. From (3.24) and (3.27) we find that

$$
\begin{aligned}
\dot{W} & =\dot{u}_{t}-\dot{z} q+\dot{\bar{z}} \bar{q} \\
& = \begin{cases}A W-2 \operatorname{Re}\left[\bar{q}^{*}(0) H_{0} q(\theta)\right], & -1 \leq \theta<0, \\
A W-2 \operatorname{Re}\left[\bar{q}^{*}(0) H_{0} q(\theta)\right], & \theta=0\end{cases} \\
& \stackrel{\text { def }}{=} A W+G(z, \bar{z}, \theta),
\end{aligned}
$$

where

$$
G(z, \bar{z}, \theta)=G_{20} \frac{z^{2}}{2}+G_{11} z \bar{z}+G_{02} \frac{\bar{z}^{2}}{2}+\cdots
$$

On the other hand, on $C_{0}$ near the origin

$$
\dot{W}=W_{z} \dot{z}+W_{\bar{z}} \dot{\bar{z}}
$$

We derive from (3.30)-(3.32) that

$$
\left(A-2 \mathrm{i} \tau_{n} \omega_{0}\right) W_{20}(\theta)=-G_{20}(\theta), \quad A W_{11}(\theta)=-G_{11}(\theta) .
$$

According to (3.29) and (3.30), we have

$$
\begin{aligned}
G(z, \bar{z}, \theta) & =-\bar{q}^{*}(0) H_{0} q(\theta)-q^{*}(0) \bar{H}_{0} q(\theta) \\
& =-g(z, \bar{z}) q(\theta)-\bar{g}(z, \bar{z}) \bar{q}(\theta), \quad-1 \leq \theta<0 .
\end{aligned}
$$

Comparing the coefficients with (3.29), we can obtain that

$$
\begin{aligned}
& G_{20}(\theta)=-g_{20} q(\theta)-\bar{g}_{02} \bar{q}(\theta), \\
& G_{11}(\theta)=-g_{11} q(\theta)-\bar{g}_{11} \bar{q}(\theta) .
\end{aligned}
$$

Substituting (3.35) into (3.33), it follows that

$$
\dot{W}_{20}(\theta)=2 \mathrm{i} \tau_{n} \omega_{0} W_{20}(\theta)+g_{20} q(\theta)+\bar{g}_{02} \bar{q}(\theta) .
$$

We can obtain that

$$
W_{20}(\theta)=\frac{\mathrm{i} g_{20} q(0)}{\tau_{n} \omega_{0}} e^{\mathrm{i} \tau_{n} \omega_{0} \theta}-\frac{\bar{g}_{02} \bar{q}(0)}{3 \mathrm{i} \tau_{n} \omega_{0}} e^{-\mathrm{i} \tau_{n} \omega_{0} \theta}+E_{1} e^{2 \mathrm{i} \tau_{n} \omega_{0} \theta} .
$$

Similarly, we have

$$
W_{11}(\theta)=\frac{g_{11} q(0)}{\mathrm{i} \tau_{n} \omega_{0}} e^{\mathrm{i} \tau_{n} \omega_{0} \theta}-\frac{\bar{g}_{11} \bar{q}(0)}{\mathrm{i} \tau_{n} \omega_{0}} e^{-\mathrm{i} \tau_{n} \omega_{0} \theta}+E_{2} e^{2 \mathrm{i} \tau_{n} \omega_{0} \theta}
$$

where

$$
E_{1}=\left(E_{1}^{(1)}, E_{1}^{(2)}, E_{1}^{(3)}\right), \quad E_{2}=\left(E_{2}^{(1)}, E_{2}^{(2)}, E_{2}^{(3)}\right) .
$$


Next we focus on the computation of $E_{1}, E_{2}$. From (3.33), we have

$$
\begin{aligned}
& \int_{-1}^{0} \mathrm{~d} \eta(\theta) W_{20}(\theta)=2 \mathrm{i} \tau_{n} \omega_{0} W_{20}(\theta)-G_{20}(\theta) \\
& \int_{-1}^{0} \mathrm{~d} \eta(\theta) W_{11}(\theta)=-G_{11}(\theta)
\end{aligned}
$$

and

$$
\begin{aligned}
& G_{20}(0)=-g_{20} q(0)-\bar{g}_{02} \bar{q}(0)+2 \tau_{n}\left(\begin{array}{c}
-\sigma c_{1} \rho_{2} \\
-c_{1} \rho_{1} \rho_{2} \\
\alpha_{2} c_{2} e^{-2 \mathrm{i} \omega_{0} \tau_{n}}\left(\sigma+\alpha_{1}\right)
\end{array}\right), \\
& G_{11}(0)=-g_{11} q(0)-\bar{g}_{11} \bar{q}(0)+2 \tau_{n}\left(\begin{array}{c}
\operatorname{Re}\left\{\rho_{2}\right\} \\
-c_{1} \operatorname{Re}\left\{\bar{\rho}_{1} \rho_{2}\right\} \\
c_{2}\left[\sigma \operatorname{Re}\left\{\alpha_{1}\right\}+\operatorname{Re}\left\{\bar{\alpha}_{1} \alpha_{2}\right\}\right]
\end{array}\right) .
\end{aligned}
$$

Substituting (3.38) and (3.42) into (3.40), then

$$
\begin{aligned}
& \left(\mathrm{i} \tau_{n} \omega_{0} I-\int_{-1}^{0} e^{\mathrm{i} \tau_{n} \omega_{0} \theta} \mathrm{d} \eta(\theta)\right) q(0)=0, \\
& \left(-\mathrm{i} \tau_{n} \omega_{0} I-\int_{-1}^{0} e^{-\mathrm{i} \tau_{n} \omega_{0} \theta} \mathrm{d} \eta(\theta)\right) \bar{q}(0)=0,
\end{aligned}
$$

we obtain

$$
\left(2 \mathrm{i} \tau_{n} \omega_{0} I-\int_{-1}^{0} e^{2 \mathrm{i} \tau_{n} \omega_{0} \theta} \mathrm{d} \eta(\theta)\right) E_{1}=2 \tau_{n}\left(\begin{array}{c}
-\sigma c_{1} \rho_{2} \\
-c_{1} \rho_{1} \rho_{2} \\
\alpha_{2} c_{2} e^{-2 \mathrm{i} \omega_{0} \tau_{n}}\left(\sigma+\alpha_{1}\right)
\end{array}\right)
$$

namely

$$
\begin{aligned}
& \left(\begin{array}{ccc}
d_{1}+\sigma \beta+2 \mathrm{i} \omega_{0} & -b_{1} & \sigma c_{1} m^{*} \\
0 & 2 \mathrm{i} \omega_{0} & c_{1} f^{*} \\
-\sigma c_{2} x^{*} e^{-2 \mathrm{i} \omega_{0} \tau_{n}} & -c_{2} x^{*} e^{-2 \mathrm{i} \omega_{0} \tau_{n}} & a+2 \mathrm{i} \omega_{0}-a e^{-2 \mathrm{i} \omega_{0} \tau_{n}}
\end{array}\right) E_{1} \\
& =2\left(\begin{array}{c}
-\sigma c_{1} \rho_{2} \\
-c_{1} \rho_{1} \rho_{2} \\
\alpha_{2} c_{2} e^{-2 \mathrm{i} \omega_{0} \tau_{n}}\left(\sigma+\alpha_{1}\right)
\end{array}\right)
\end{aligned}
$$

Similarly, we have

$$
-\left(\begin{array}{ccc}
-d_{1}-\sigma \beta & b_{1} & -\sigma c_{1} m^{*} \\
0 & 0 & -c_{1} f^{*} \\
-\sigma c_{2} x^{*} & -c_{2} x^{*} & 0
\end{array}\right) E_{2}=2\left(\begin{array}{c}
-\sigma \operatorname{Re}\left\{\rho_{2}\right\} \\
-c_{1} \operatorname{Re}\left\{\bar{\rho}_{1} \rho_{2}\right\} \\
c_{2}\left[\sigma \operatorname{Re}\left\{\alpha_{1}\right\}+\operatorname{Re}\left\{\bar{\alpha}_{1} \alpha_{2}\right\}\right]
\end{array}\right) .
$$

Therefore, $E_{1}$ and $E_{2}$ can be determined from (3.44) and (3.45). Then $g_{21}$ can be determined by the parameters and delay. Thus, we can compute the following quantities:

$$
C_{1}(0)=\frac{\mathrm{i}}{2 \tau_{n} \omega_{0}}\left(g_{20} g_{11}-2\left|g_{11}\right|^{2}-\frac{\left|g_{02}\right|^{2}}{2}\right)+\frac{g_{21}}{2},
$$




$$
\begin{aligned}
& \mu_{2}=-\frac{\operatorname{Re}\left\{C_{1}(0)\right\}}{\operatorname{Re}\left\{\lambda^{\prime}\left(\tau_{n}\right)\right\}}, \quad \beta_{2}=2 \operatorname{Re}\left\{C_{1}(0)\right\}, \\
& T_{2}=-\frac{\operatorname{Im}\left\{C_{1}(0)\right\}+\mu_{2} \operatorname{Im}\left\{\lambda^{\prime}\left(\tau_{n}\right)\right\}}{\omega_{0}} .
\end{aligned}
$$

Theorem 3.2 (i) $\mu_{2}$ determines the direction of the Hopf bifurcation. If $\mu_{2}>0(<0)$, then the Hopf bifurcation is supercritical (subcritical), and the bifurcating periodic solution exists for $\tau>\tau_{0}\left(\tau<\tau_{0}\right)$;

(ii) $\beta_{2}$ determines the stability of bifurcating periodic solutions. If $\beta_{2}>0(<0)$, the bifurcating periodic solutions are unstable (stable);

(iii) $T_{2}$ determines the period of bifurcating periodic solutions. If $T_{2}>0(<0)$, the period increases (decreases).

\section{Numerical simulation}

Example 1 Let $b_{1}=3, d_{1}=0.1, c_{1}=2, c_{2}=0.5, \beta=2, a=0.3$, i.e., we consider the following ODE system:

$$
\left\{\begin{array}{l}
m^{\prime}(t)=3 f(t)-0.1 m(t)-2 \sigma m(t) x(t), \\
f^{\prime}(t)=f(t)(\beta-2 x(t)), \\
x^{\prime}(t)=x(t)(-0.3+0.5 \sigma m(t)+0.5 f(t)) .
\end{array}\right.
$$

From Theorem 2.1 we know $\sigma=\sigma_{0}=1$ is the critical value for the Hopf bifurcation. When $\sigma=1.2$, the positive equilibrium $E^{\prime \prime}=(0.2951,0.2459,1)$ of system $(4.1)$ is locally asymptotically stable (see Figure 1); when $\sigma=0.9$, there exists a nontrivial orbitally periodic orbit of system (4.1). From the Hopf bifurcation analysis in Section 2.2 and the numerical result $l_{1}=-0.4047<0$, the Hopf bifurcating periodic solution is stable (see Figure 2). A typical strange attractor appears when $\sigma=0.35$ (see Figure 3).
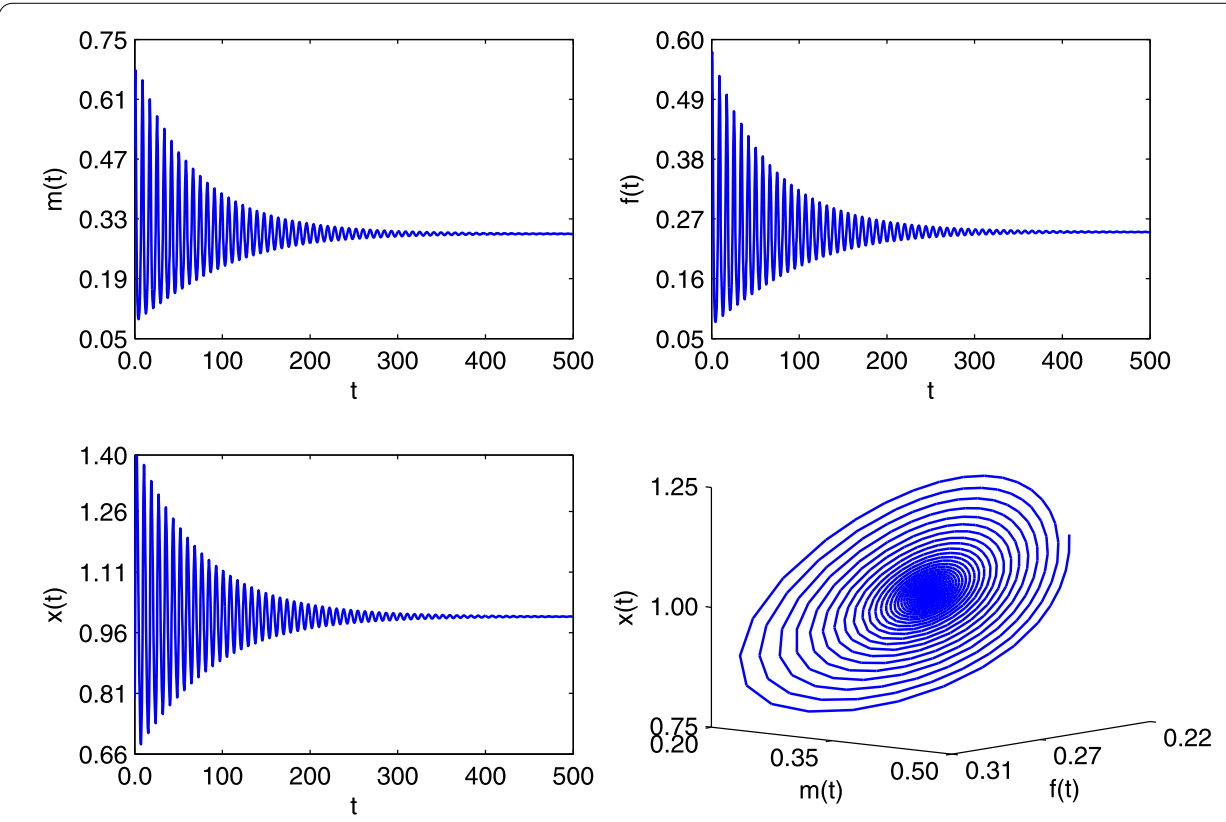

Figure 1 The positive equilibrium $E^{*}=(0.2951,0.2459,1)$ of system $(4.1)$ is locally asymptotically stable when $\sigma=1.2>\sigma_{0}$. 

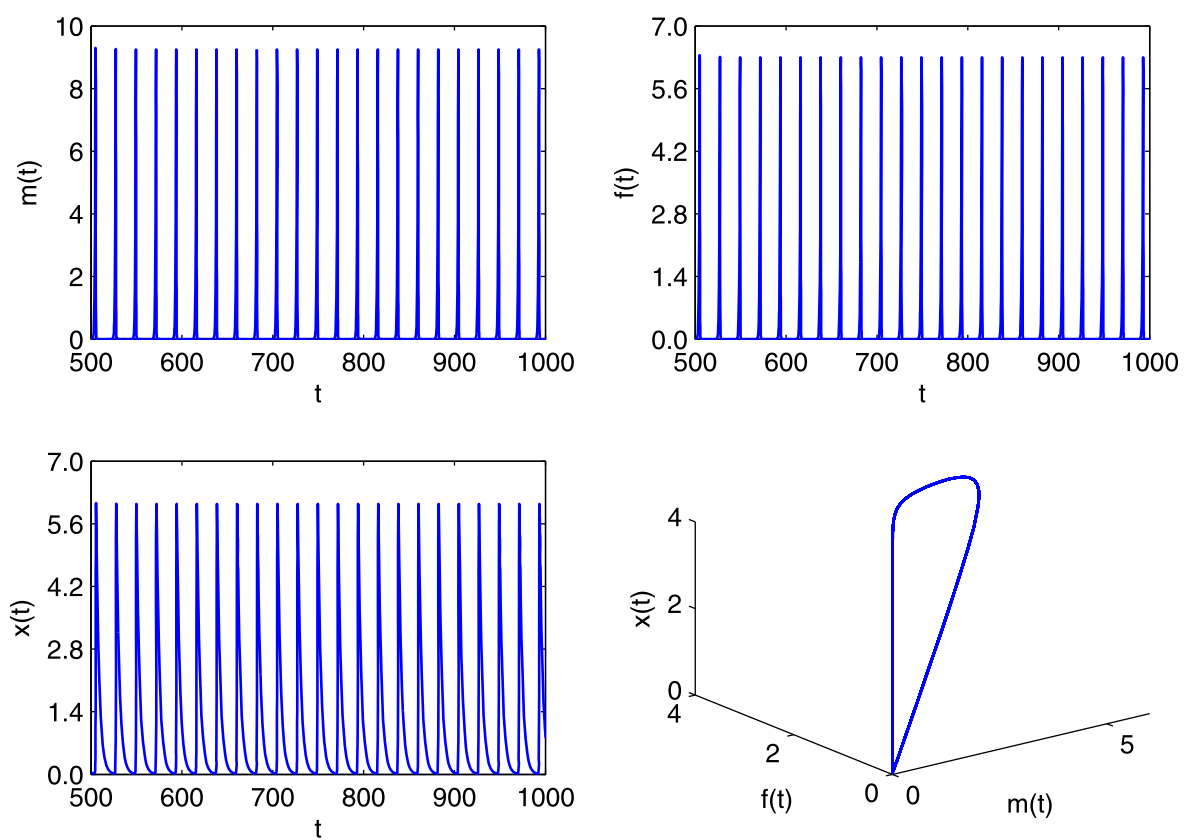

Figure 2 Stable Hopf bifurcating periodic solutions from $E^{*}=(0.3913,0.2478,1)$ of system $(4.1)$ when $\sigma=0.9<\sigma_{0}$.
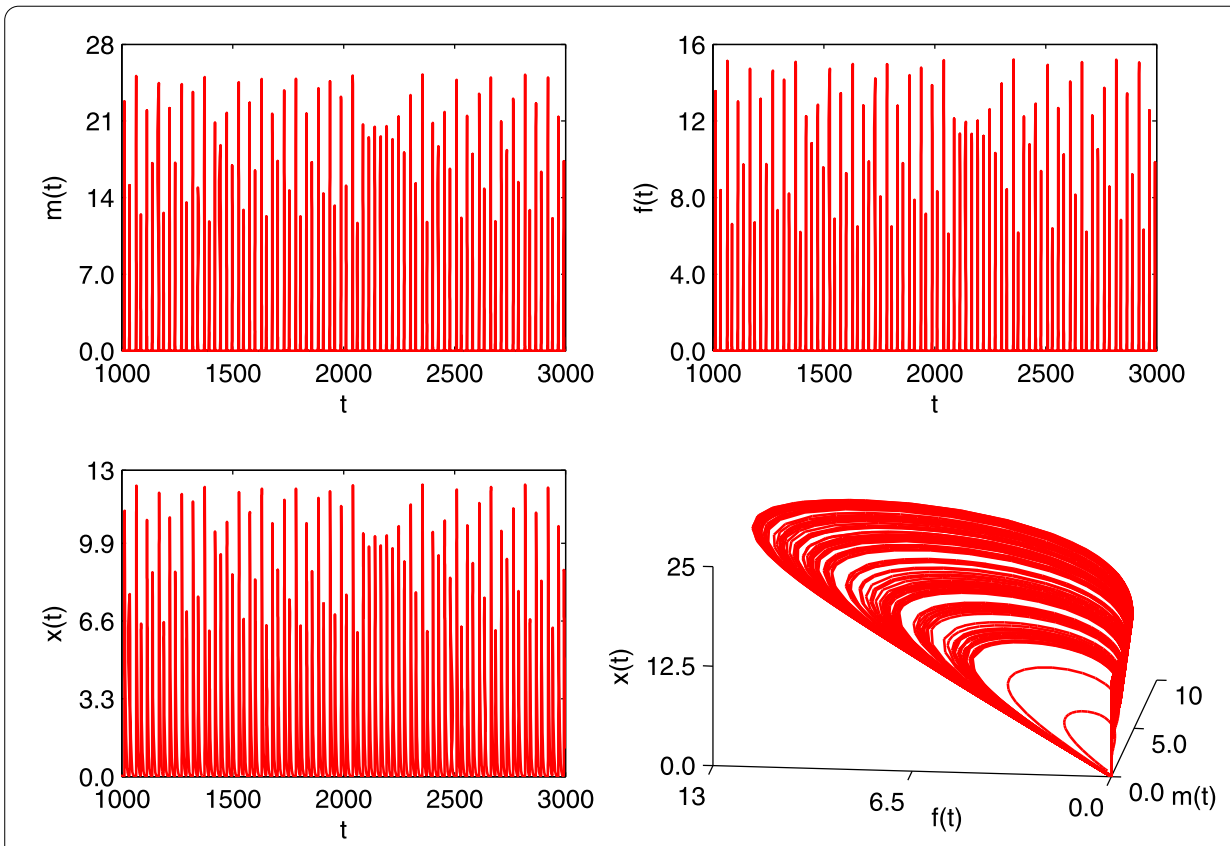

Figure 3 The strange attractor of system (4.1) when $\sigma=0.35$. 

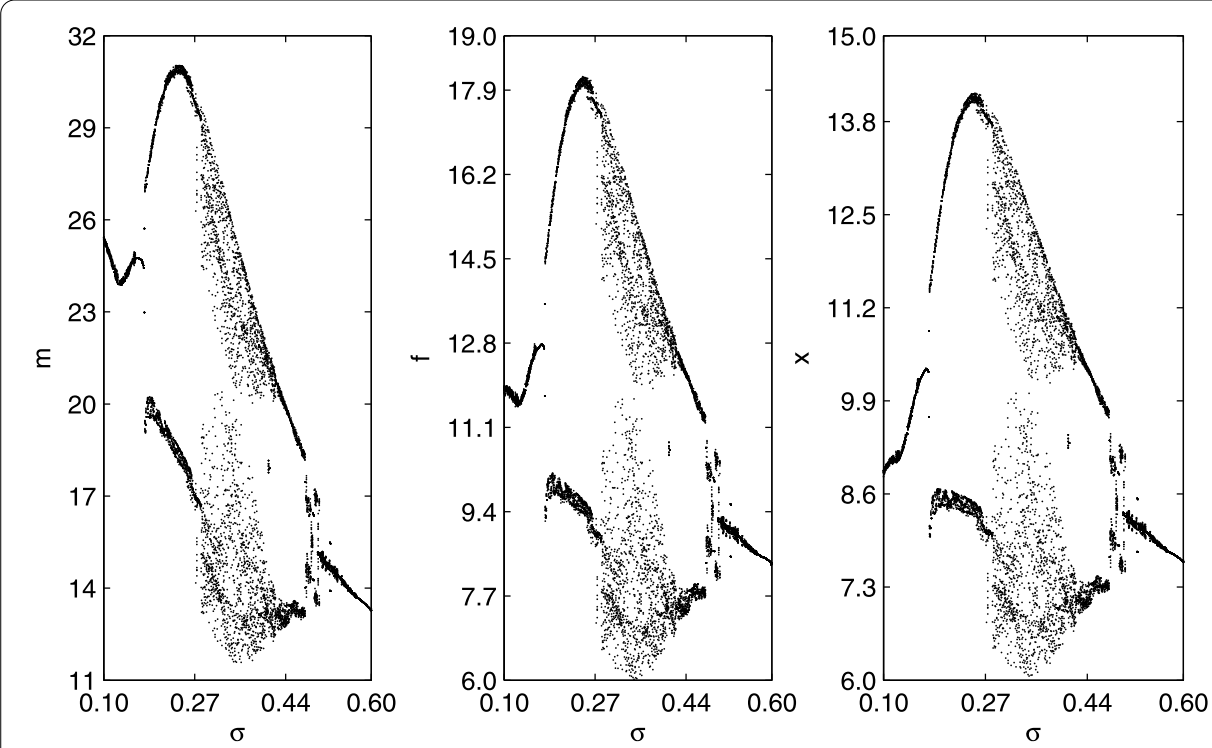

Figure 4 Bifurcation diagrams of system (4.1) for $\sigma$ over $[0.1,0.6]$ show the effect of sex ratio coefficient $\sigma$ on the dynamic behavior.

Furthermore, we also investigate the effect of the sex ratio $\sigma$ on system (4.1). The bifurcation diagrams of $\sigma$ over [0.1,0.6] show that system (4.1) has rich dynamics (see Figure 4), including (1) periodic oscillating, (2) period-doubling bifurcations, (3) periodhalving bifurcations and (4) chaos. When $0.1 \leq \sigma<\sigma_{1} \approx 0.176$, system (4.1) experiences a $T$-periodic solution (Figure 5(a)). When $\sigma>\sigma_{1}$, the $T$-periodic solution leads to a $2 T$-periodic solution, and there is a period-doubling bifurcation leading to chaos when $\sigma>\sigma_{2} \approx 0.284$ (Figure 5(b), (c)). When $\sigma>\sigma_{3} \approx 0.426$, the chaos suddenly disappears and a $2 T$-periodic solution appears, and there is a cascade of period-halving bifurcations leading to a $T$-periodic solution when $\sigma_{3}<\sigma \leq 0.6$ (Figure 5(c)-(e)).

Example 2 Let $b_{1}=3, d_{1}=0.4, \sigma=2.2, c_{1}=1.5, c_{2}=0.4, \beta=1.6, a=0.5$, we consider the following DDE system:

$$
\left\{\begin{array}{l}
m^{\prime}(t)=3 f(t)-0.4 m(t)-3.3 m(t) x(t), \\
f^{\prime}(t)=f(t)(1.6-1.5 x(t)), \\
x^{\prime}(t)=-0.5 x(t)+0.88 m(t-\tau) x(t-\tau)+0.4 f(t-\tau) x(t-\tau) .
\end{array}\right.
$$

System (4.2) has a unique positive equilibrium point $E^{*}=(0.3565,0.4658,1.0667)$. From the results in Section 3, we evaluate that $p=15.3664, q=0.3945, r=-9.8345$, $\omega_{0}=0.8767, \tau_{0}=0.1818$, and the positive equilibrium point $E^{\prime \prime}$ is asymptotically stable when $\tau \in\left[0, \tau_{0}\right)=\left[0,0.1818\right.$ ) (see Figure 6 ) and unstable when $\tau>\tau_{0}$.

By the theory of Hassard [30], as it has been discussed in the previous section, we may also determine the direction of the Hopf bifurcation and the other properties of bifurcating periodic solutions. From the formulae in Section 3 we compute the values of $\mu_{2}, \beta_{2}$, and $T_{2}$ as

$$
\mu_{2}=-6.5132<0, \quad \beta_{2}=1.2053>0, \quad T_{2}=4.0722>0,
$$




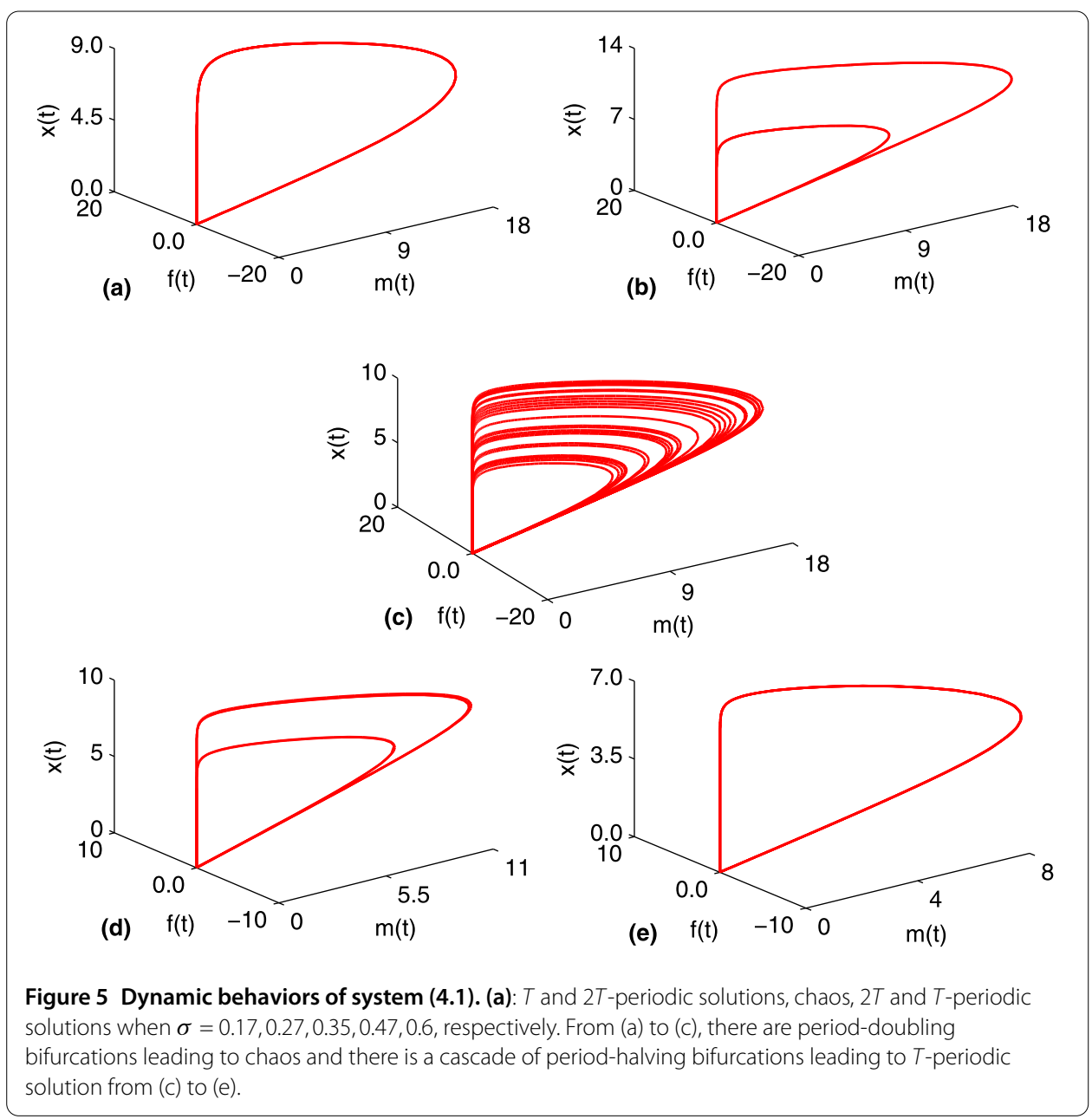

from which we conclude that since $\mu_{2}<0$, the Hopf bifurcation of system (4.2) occurring at $\tau_{0}=0.1818$ is subcritical and the bifurcating periodic solution exists when $\tau$ crosses $\tau_{0}$ to the left; also since $\beta_{2}>0$, the bifurcating periodic solution is unstable (see Figure 7).

Example 3 We consider ODE system (4.1) with time delays:

$$
\left\{\begin{array}{l}
m^{\prime}(t)=3 f(t)-0.1 m(t)-2 \sigma m(t) x(t) \\
f^{\prime}(t)=f(t)(\beta-2 x(t)) \\
x^{\prime}(t)=-0.3 x(t)+0.5 \sigma m(t-\tau) x(t-\tau)+0.5 f(t-\tau) x(t-\tau)
\end{array}\right.
$$

From Example 1 we know that the positive equilibrium $E^{*}=(0.2951,0.2459,1)$ of system (4.3) is locally asymptotically stable when $\tau=0$, and there exists a critical value $\tau_{0} \approx 0.045$ such that system (4.3) experiences the Hopf bifurcation. That is to say, time delay would make the locally asymptotically stable $E^{*}$ of ODE system (4.1) unstable if we increase the time delay to some critical value, and a typical periodic oscillation is observed when $\tau=$ 0.042 (see Figure 8 , from left closed to $\tau_{0} \approx 0.045$ ). The stable Hopf bifurcating periodic solution of system (4.1) for $\sigma=0.9$ would be destroyed, even for a very small time delay, and a typical unstable periodic oscillation appears when $\tau=0.001$ for system (4.3). The amplitude of the oscillation is increased with the increasing of the time $t$ (see Figure 9). 

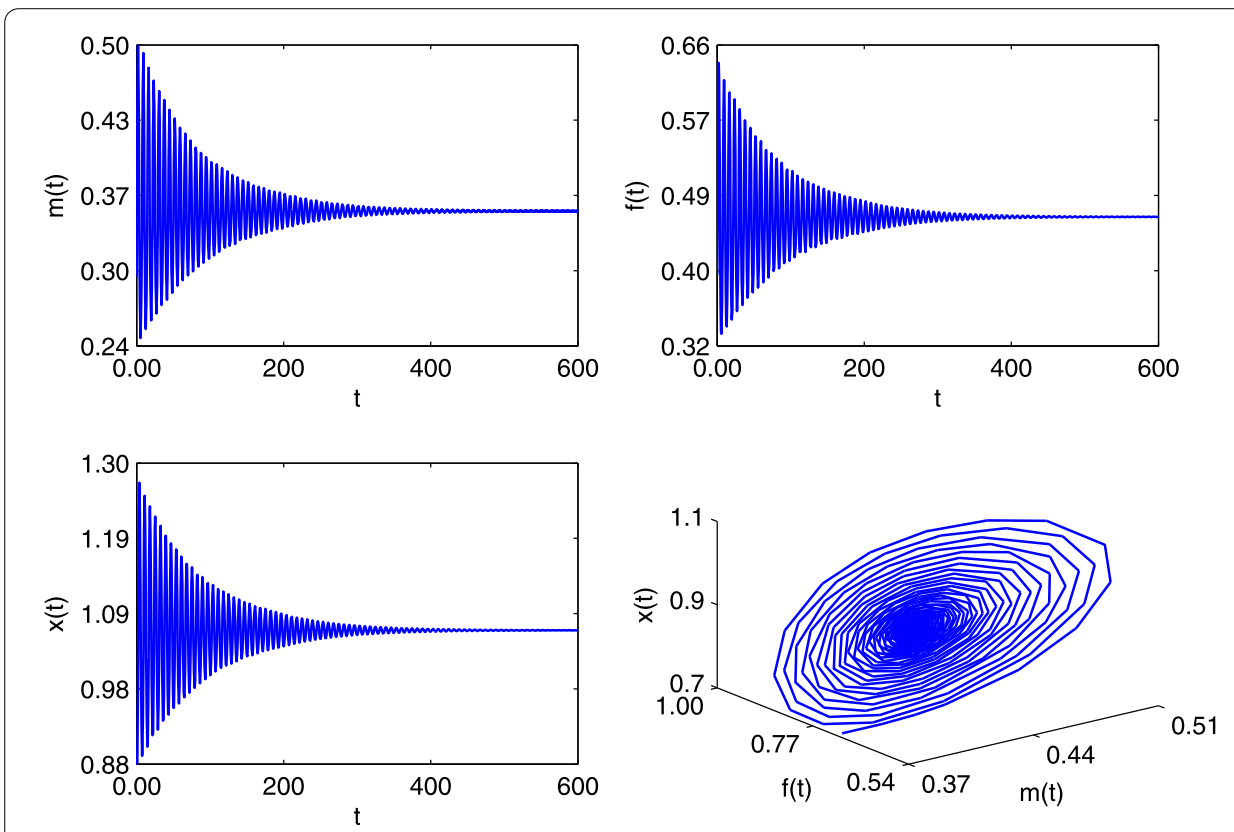

Figure 6 The positive equilibrium $E^{*}=(0.3565,0.4658,1.0067)$ of system $(4.2)$ is locally asymptotically stable when $\tau=0.15<\tau_{0}=0.1818$.
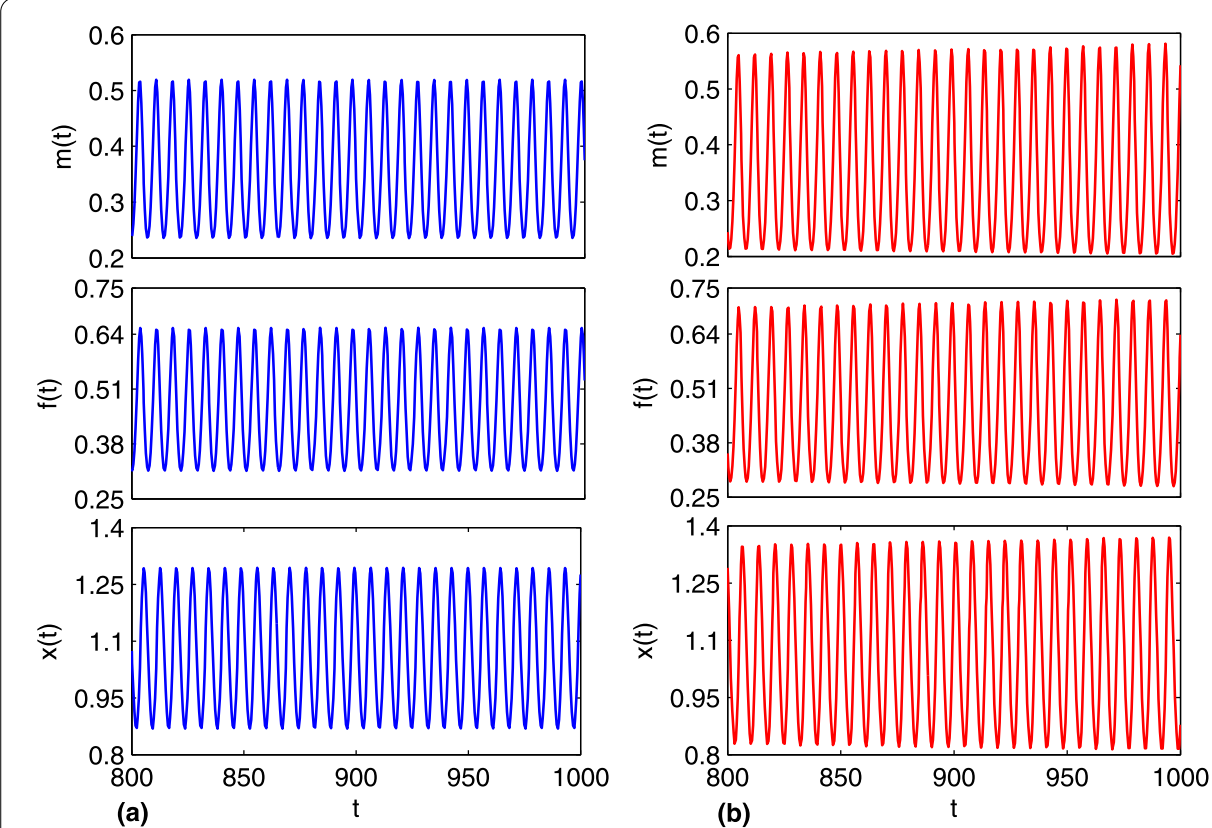

Figure 7 Hopf bifurcating periodic solutions of system (4.2) when $\tau=0.181<\tau_{0}$ and $\tau=0.187$ near $\tau_{0}$. 

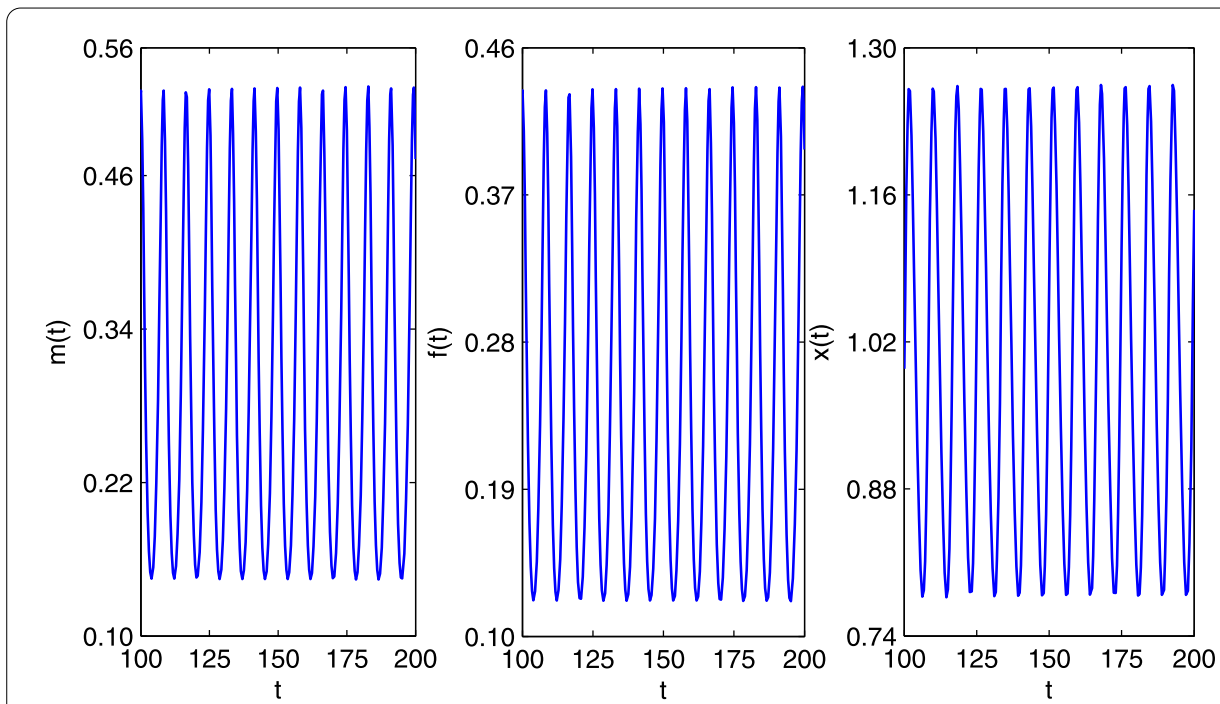

Figure 8 Stable periodic oscillations about $E^{*}=(0.3913,0.2478,1)$ of system $(4.3)$ when $\sigma=0.9$ and $\tau=0.042$.

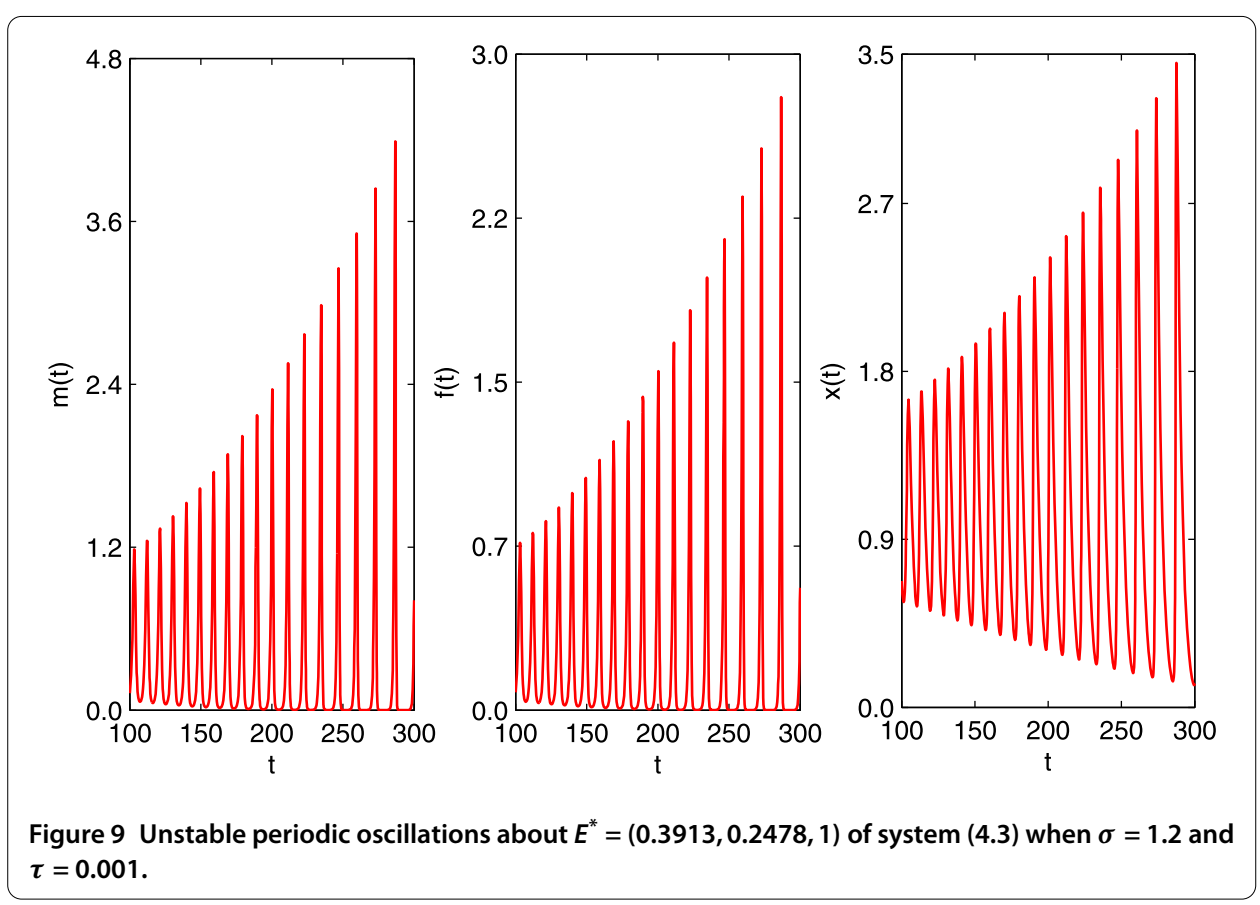

When $\sigma=0.35$, a typical unbounded oscillation solution is observed for a very small time delay $\tau=0.001$ and time $t \approx 101$ (see Figure 10). That is to say, a very small delay would make DDE system (4.3) extinct (unbounded oscillation) undergoing a series of fast-slow oscillations and destroying the permanence of it, if the corresponding ODE system (4.1) is chaotic oscillating when $\sigma=0.35$. All the analysis shows that the time delay would destroy the stability of the system, even make the system die out. 

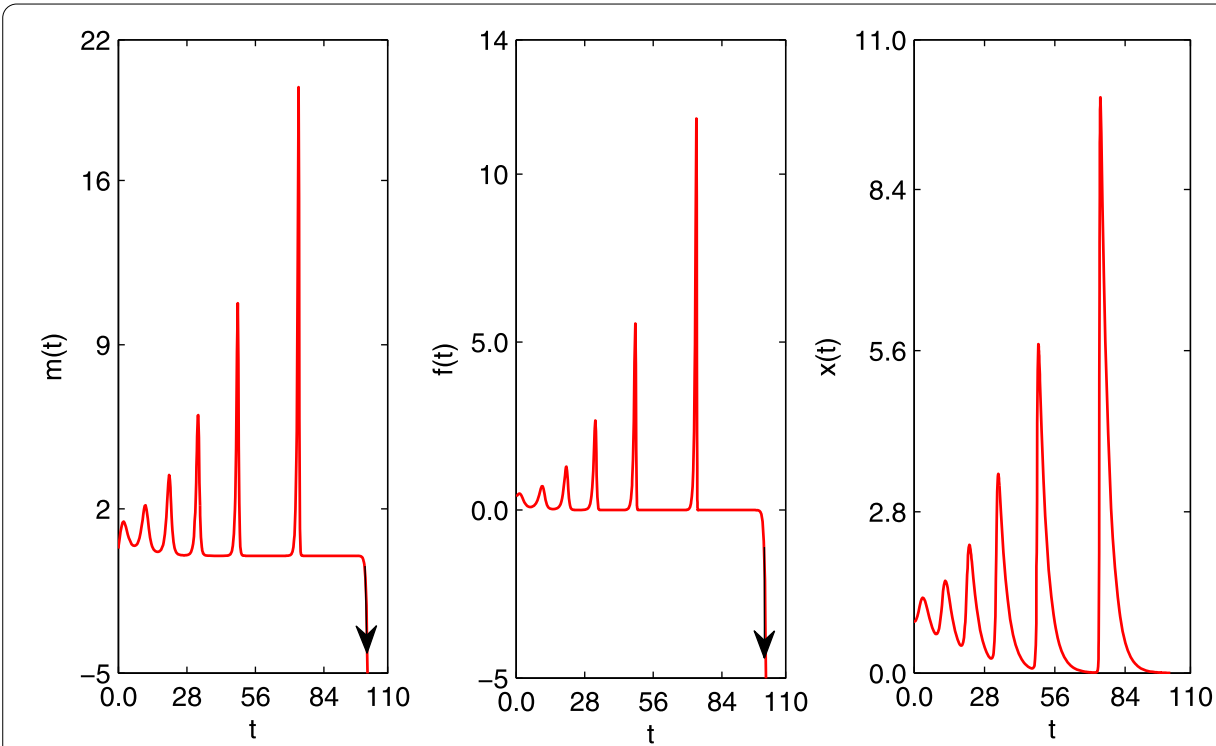

Figure 10 The solutions $m(t), f(t)$ of prey populations tend to $-\infty$ (unbounded solutions) when $\sigma=0.35$ and $\tau=0.001$.

\section{Conclusion}

In this paper, we have investigated a predator-prey system with sex-structure and sexual favoritism. Firstly, the impact of the sexual favoritism coefficient $\sigma$ on the stability of the ODE model is studied. From Theorem 2.1, we know the sexual favoritism coefficient $\sigma$ would determine the stability of ODE system (1.2). In the ecology, sexual favoritism predation could impact population dynamics differently and affect reduced male and female densities in the prey. The numerical simulations show that ODE system (1.2) has complicated dynamic behaviors when we change the parameter $\sigma$, including periodic oscillating, period-doubling bifurcations, period-halving bifurcations and chaos. That is to say, sexual favoritism coefficient $\sigma$ would be an important factor to affect the dynamic behaviors of the system. Secondly, by analyzing the associated characteristic equation, the impact of the time delay $\tau$ on the stability of DDE system (1.3) is obtained and the explicit formulae, which determine the stability, direction, and other properties of bifurcating periodic solutions, are also obtained by the Hassard method.

We have obtained estimated length of gestation delay which does not affect the stable coexistence of both predator and prey species at their equilibrium values. From the numerical simulations, we know that the Hopf bifurcation is subcritical and the bifurcating periodic solutions are unstable. It is clear that the larger values of gestation time delay cause fluctuation in population density, and even a very small time delay would make the system subject to unstable oscillation and extinct. These are harmful delays. How to control the bifurcation arising from the DDE system? How can one do this if the time delays make the system subject to unbounded oscillations? The time-varying control strategies and the impulsive control strategies would be considered [35], which could both improve the stability of the system and control the amplitude of the bifurcated periodic solution effectively. We will continue to study these problems in the future. 
Authors' contributions

SL carried out the main part of this manuscript. ZX participated in the discussion and gave the examples. All authors read and approved the final manuscript.

\section{Author details}

'Department of Mathematics, Qiannan Normal College for Nationalities, Duyun, Guizhou 558000, China. ${ }^{2}$ Department of Mathematics, Nanchang University, Nanchang, Jiangxi 330031, China.

\section{Acknowledgements}

The authors would like to thank the reviewers and the editor for their valuable suggestions and comments which have led to a much improved paper. This work was supported by the Natural Science Foundation of Guizhou Province (No. [2011]2116), Natural Science Foundation of Jiangxi Province (No. 20122BAB201002)

Received: 29 November 2012 Accepted: 18 June 2013 Published: 23 July 2013

\section{References}

1. Hamilton, WD: Extraordinary sex ratios. A sex-ratio theory for sex linkage and inbreeding has new implications in cytogenetics and entomology. Science 156(3774), 477-488 (1967)

2. Clark, AB: Sex ratio and local resource competition in a prosimian primate. Science 201(4351), 163-165 (1978)

3. Wakano, JY: Evolution of extraordinary female-biased sex ratios: the optimal schedule of sex ratio in local mate competition. J. Theor. Biol. 237(2), 193-202 (2005)

4. James, WH: Possible constraints on adaptive variation in sex ratio at birth in humans and other primates. J. Theor. Biol. 238(2), 383-394 (2006)

5. Hu, XS, Yeh, FC, He, F: Sex-ratio distortion driven by migration loads. Theor. Popul. Biol. 72(4), 547-559 (2007)

6. Law, PR, Linklater, WL: Optimising the sex ratio of translocation for genetic rescue as a function of invested resources. Ecol. Model. 208(2-4), 317-341 (2007)

7. Onagbola, EO, Fadamiro, HY, Mbata, GN: Longevity, fecundity, and progeny sex ratio of Pteromalus cerealellae in relation to diet, host provision, and mating. Biol. Control 40(2), 222-229 (2007)

8. Kamimura, Y, Abe, J, Ito, $\mathrm{H}$ : The continuous public goods game and the evolution of cooperative sex ratios. J. Theor. Biol. 252(2), 277-287 (2008)

9. Wang, $X Y$, Yang, $Z Q, W u, H$, et al.: Effects of host size on the sex ratio, clutch size, and size of adult Spathius agrili, an ectoparasitoid of emerald ash borer. Biol. Control 44(1), 7-12 (2008)

10. Charnov, EL: The Theory of Sex Allocation. Princeton University Press, Princeton (1982)

11. Liu, HW, Wang, RX, Liu, JX: The prey-predator model with sex-structure. J. Biomath. 20(2), 179-182 (2005) (in Chinese)

12. Boukal, DS, Berec, L, Křivan, V: Does sex-selective predation stabilize or destabilize predator-prey dynamics? PLoS ONE 3(7), e2687 (2008)

13. Long, SJ: Attracting and invariant sets of nonlinear neutral differential equations with delays. Adv. Differ. Equ. 2012, $113(2012)$

14. Lu, HY, Wang, WG: Dynamics of a delayed discrete semi-ratio-dependent predator-prey system with Holling type IV functional response. Adv. Differ. Equ. 2011, 7 (2011)

15. Zhang, QH, Yang, LH, Liao, DX: Existence and globally exponential stability of equilibrium for fuzzy BAM neural networks with distributed delays and impulse. Adv. Differ. Equ. 2011, 8 (2011)

16. Alzabut, JO: Existence of periodic solutions for a type of linear difference equations with distributed delay. Adv. Differ Equ. 2012, 53 (2012)

17. $\mathrm{Wu}, \mathrm{RC}$, Liu, JL: Isochronal function projective synchronization between chaotic and time-delayed chaotic systems. Adv. Differ. Equ. 2012, 37 (2012)

18. Sangapate, P: New sufficient conditions for the asymptotic stability of discrete time-delay systems. Adv. Differ. Equ. 2012, 28 (2012)

19. Dong, YL, Wei, J: Output feedback stabilization of nonlinear discrete-time systems with time-delay. Adv. Differ. Equ. 2012, 73 (2012)

20. Chen, HB, Tang, HW, Sun, JT: Periodic solutions of second-order differential equations with multiple delays. Adv. Differ. Equ. 2012, 43 (2012)

21. Zhao, YX, Ma, YC: Stability of neutral-type descriptor systems with multiple time-varying delays. Adv. Differ. Equ. 2012, 15 (2012)

22. Song, QK, Cao, JD: Synchronization of nonidentical chaotic neural networks with leakage delay and mixed time-varying delays. Adv. Differ. Equ. 2011, 16 (2011)

23. Wang, WD, Chen, LS: A predator-prey system with stage-structure for predator. Comput. Math. Appl. 33(8), 83-91 (1997)

24. Xiao, YN, Chen, LS: Modeling and analysis of a predator-prey model with disease in the prey. Math. Biosci. 171(1), 59-82 (2001)

25. Zhao, T, Kuang, Y, Smith, HL: Global existence of periodic solutions in a class of delayed Gause-type predator-prey systems. Nonlinear Anal., Theory Methods Appl. 28(8), 1373-1394 (1997)

26. Xiong, XS, Zhang, ZQ: Existence and global attractivity of a periodic solution for a prey-predator model with sex-structure. Appl. Math. Comput. 190(2), 1213-1224 (2007)

27. Li, BW, Xiong, XS: Existence and global attractivity of periodic solution for a discrete prey-predator model with sex structure. Nonlinear Anal., Real World Appl. 11(3), 1986-2000 (2010)

28. Pei, YZ, Yang, Y, Li, CG, et al.: Pest management of a prey-predator model with sexual favoritism. Math. Med. Biol. 26(2), 97-115 (2009)

29. Kuznetsov, YA: Elements of Applied Bifurcation Theory, 2nd edn. Springer, New York (1998)

30. Hassard, BD, Kazarinoff, ND, Wan, YH: Theory and Applications of Hopf Bifurcation. Cambridge University Press, Cambridge (1981)

31. Liu, WM: Criterion of Hopf bifurcation without using eigenvalues. J. Math. Anal. Appl. 182(1), 250-255 (1994) 
32. Hale, JK: Theory of Functional Differential Equations. Springer, New York (1997)

33. Freedman, HI, Rao, VSH: The trade-off between mutual interference and time lags in predator-prey systems. Bull. Math. Biol. 45(6), 991-1004 (1983)

34. Erbe, LH, Freedman, HI, Rao, VSH: Three-species food chain models with mutual interference and time delays. Math. Biosci. 80(1), 57-80 (1986)

35. Zhao, YH, Yu, XH, Wang, L: Bifurcation and control in an inertial two-neuron system with time delays. Int. J. Bifurc. Chaos 22(2), $1250036(2012)$

doi:10.1186/1687-1847-2013-219

Cite this article as: Li and Xiong: Bifurcation analysis of a predator-prey system with sex-structure and sexual favoritism. Advances in Difference Equations 2013 2013:219.

Submit your manuscript to a SpringerOpen ${ }^{\circ}$ journal and benefit from:

- Convenient online submission

- Rigorous peer review

- Immediate publication on acceptance

- Open access: articles freely available online

- High visibility within the field

- Retaining the copyright to your article 